\title{
Chemical controllable gene drive in Drosophila
}

Dongwoo Chae ${ }^{1 *}, 7$, Junwon Lee ${ }^{4,7}$, Nayoung Lee ${ }^{2,7}$, Kyungsoo Park ${ }^{1}$, Seok Jun Moon ${ }^{2 *}$, and Hyongbum Henry Kim ${ }^{1,3,5,6^{*}}$

${ }^{1}$ Department of Pharmacology, BK21 PLUS Project for Medical Science, Yonsei University College of Medicine, 50-1Yonsei-ro, Seodaemun-gu, Seoul 03722, Korea

${ }^{2}$ Department of Oral Biology, BK21 PLUS Project, Yonsei University College of Dentistry, 50-1 Yonsei-ro, Seodaemun-gu, Seoul 03722, Korea

${ }^{3}$ Center for Nanomedicine, Institute for Basic Science (IBS), 50 Yonsei-ro, Seodaemun-gu, Seoul 03722, Korea

${ }^{4}$ Department of Ophthalmology, Institute of Vision Research, Yonsei University College of Medicine, 50-1 Yonsei-ro, Seodaemun-gu, Seoul 03722, Korea

${ }^{5}$ Graduate Program of Nano Biomedical Engineering, Advanced Science Institute, Yonsei University, Seoul, Republic of Korea.

${ }^{6}$ Severance Biomedical Science Institute, Yonsei University College of Medicine, Seoul,

Republic of Korea

${ }^{7}$ These authors contributed equally to this work.

Lead Contact: Dongwoo Chae, email: dongy@yuhs.ac *Correspondence: e-mail-dongy@yuhs.ac or sjmoon@yuhs.ac or HKIM1@yuhs.ac 
A Junction $\mathrm{PCR}$ primer pairs

Out-in junction: Out-in F + Out-in R

In-out junction: In-out $F+$ In-out $R$

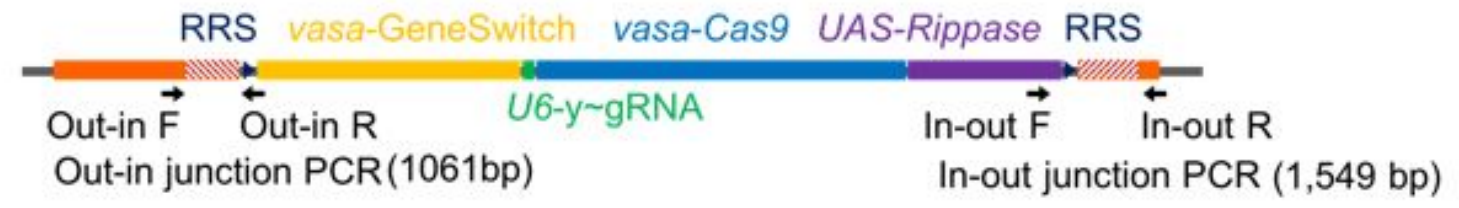

B Absence $\mathrm{PCR}$ primer pair: $\mathrm{Y}$ anterior $+\mathrm{Y}$ posterior

-In the absence of CGD cassette (expected amplicon size: 716 bp)

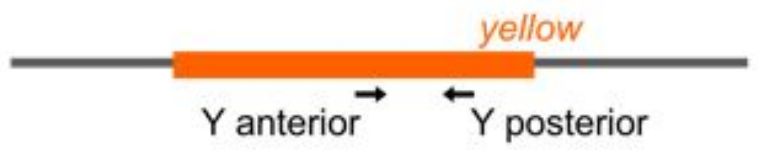

-In the presence of CGD cassette (expected amplicon size: 15,507 bp or no band)

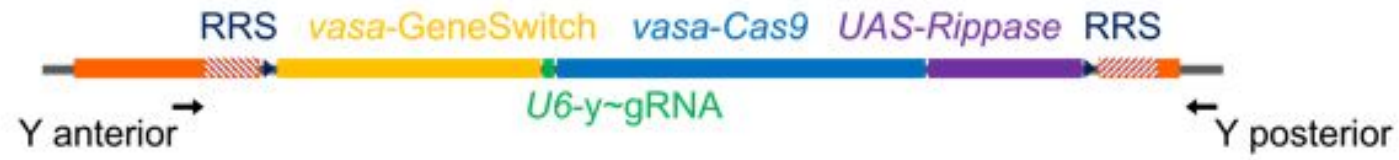

-With removal of CGD cassette by Rippase-mediated recombination (expected amplicon size: 1,116 bp)

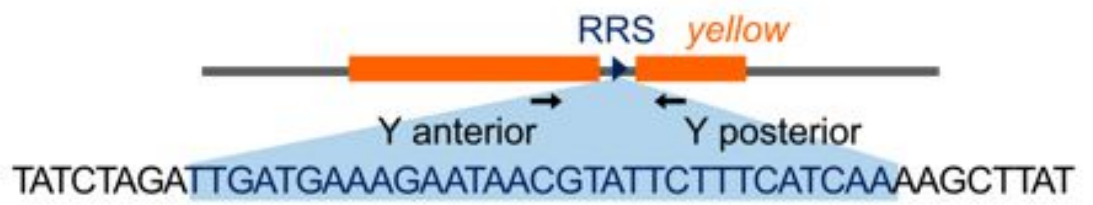

Figure S1. PCR-based evaluation of controllable gene drive (CGD) in Drosophila. Diagram of the CGD construct: Two $y$ locus homology arms flanking the vasa-GeneSwitch, $2 \times U A S-R i p p a s e$, vasaCas9, U6-y gRNA, and two Rippase recognition site (RRS) transgenes are shown. The locations of regions targeted by PCR primers used for the evaluation of CGD element insertion are indicated (the sequences of primers are shown in Table S1). (A) To confirm that the cassette integrated correctly into the target site on the genome, PCRs amplifying the junctional regions were performed on both sides of the cassette. This is referred to as "junction PCR," which includes "in-out junction PCR" and "out-in junction PCR." (B) A further PCR reaction was performed to check for the absence of an insert between HA1 and HA2, which includes no change at the flanked site or introduction of small indels. This is referred to as "insert absence PCR" (or, for brevity, absence PCR). The expected amplicon sizes generated using out-in and in-out junction PCRs were 1,061 bp (with Vg341/HA1 R1 primers) and 1,549 bp (with yellow $R$ 4387/Rippase P F primers), respectively. The expected amplicon size generated using absence PCR was 716 bp (with $Y$ anterior/Y posterior primers). Rippase recognition site sequence: TTGATGAAAGAATAACGTATTCTTTCATCAA is highlighted. 


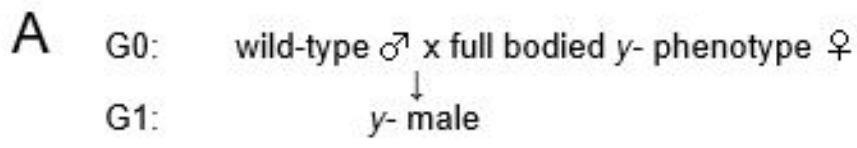

\begin{tabular}{|c|c|c|c|c|}
\hline \multirow[t]{2}{*}{ Progeny } & \multirow{2}{*}{$\begin{array}{l}\text { PCR analysis of G1 } \\
\text { (junction): (absence) }\end{array}$} & \multicolumn{2}{|c|}{ Founders } & \multirow[t]{2}{*}{ Total } \\
\hline & & $y-\mathrm{G} 0-1 \%$ & $y$ - G0-2 우 & \\
\hline \multirow[t]{3}{*}{ G1 ot } & $(+):(-)$ & $90 \%(9 / 10)$ & $100 \%(10 / 10)$ & $95 \%(19 / 20)$ \\
\hline & $(+):(+)$ & & & \\
\hline & $(-):(+)$ & $10 \%(1 / 10)$ & & $5 \%(1 / 20)$ \\
\hline \multirow[t]{3}{*}{ G1 우 } & $(+):(-)$ & & & \\
\hline & $(+):(+)$ & $100 \%(9 / 9)$ & $100 \%(7 / 7)$ & $100 \%(16 / 16)$ \\
\hline & $(-):(+)$ & & & \\
\hline
\end{tabular}

C

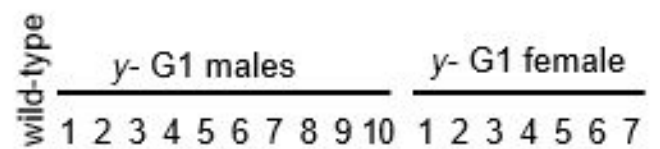

Lanes M 123456789101112131415161718

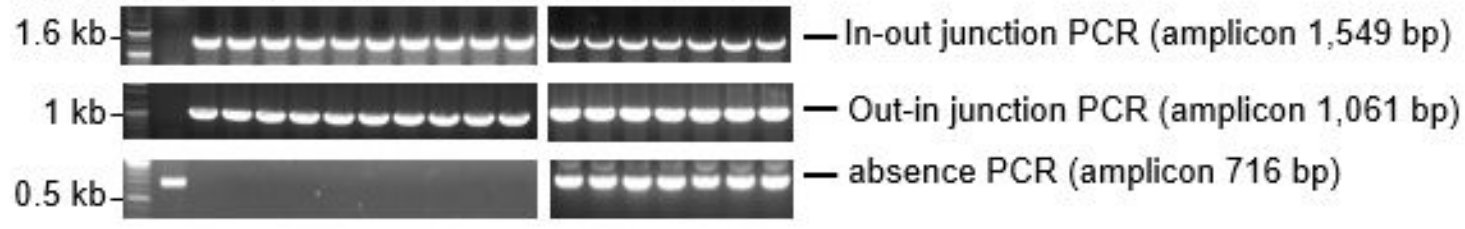

$\mathrm{D}$

GGTITTGGACACTGGAACCG

yellow GTGGTCGGCTGTGGGTITIGGACACTGGAACCGTGGGCATCGG

NHEJ 1 GTGGTCGGCTGTGGGTITTGACA - . . . - - CGTGGGCATCGG

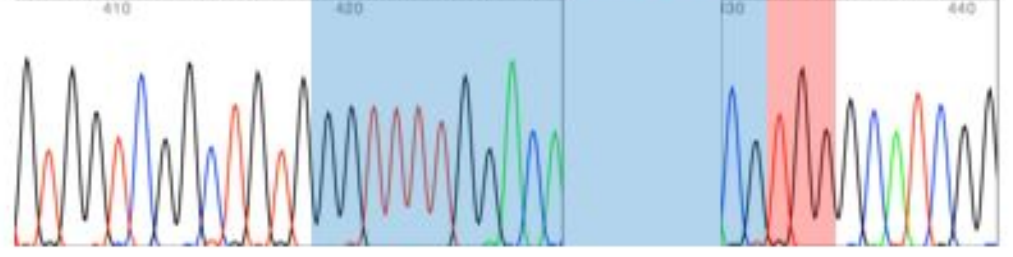

Figure S2. Characterization of two controllable gene drive (CGD) founders using progeny testing. (A) Two female founders, named y- G0-1 and y- G0-2, were established through embryo injection of a plasmid containing the CGD cassette and homologous arms (HA1 and HA2) described in Figure 1A. G1 generation flies were obtained when the two founders were crossed with the wild-type males. (B) Using the genomic DNA of G1 individuals, junction and absence PCRs were performed. In all cases, there was no discrepancy in the results between in-out junction PCR and out-in junction PCR. When there were "junction bands" and no "absence bands," we assumed that gene drive had occurred. When there were both "junction bands" and "absence bands," flies were judged to be molecularly mosaic for CGD. If there were no "junction bands" and only an "absence band," the result was interpreted as indicating no change in the genome or that a small indel had occurred near the cleavage site by nonhomologous end joining (NHEJ). In the case of $\mathrm{G} 1$ males, $90 \%$ or $100 \%$ of cases were considered to show full gene drive with "junction PCR" (+) and "absence PCR" (-). There were no cases with "junction 
PCR" (+) and "absence PCR" (+). There was also one case with "junction PCR" (-) and "absence PCR" $(+)$, which was presumed to indicate no change or a small indel through NHEJ. In all G1 females, "junction PCR" (+) and "absence PCR" (+) were observed, and thus these females were assumed to be molecularly mosaic. In a previous study, all females harboring a gene drive cassette were observed to be molecularly mosaic ${ }^{1}$. (C) Representative gel electrophoresis. PCR analysis of a wild-type fly (lane 1), y- G1 males (lanes 2-11), and y- G1 females (lanes 12-18). The full-bodied y- phenotype G1 males (carrying a single X-chromosome) display only gene drive-derived PCR products (lanes 2-11), fullbodied $y$ - phenotype $\mathrm{G} 1$ females showed mosaicism, being positive for both junction and absence PCRs. (D) An example of the sequence for CGD-induced indels. gRNA target sites were PCR amplified and sequenced from y- males with "junction PCR" (-) and "absence PCR" (+). A 7-bp deletion around the target cleavage site produces a non-functional frame-shift. 


\section{A G1: wild-type $\sigma^{\top} x$ full bodied $y$ - phenotype 우 G2: wild-type $\sigma^{7} \mathrm{x}$ full bodied $y$-phenotype 우 G3: progeny phenotype analysis}

B

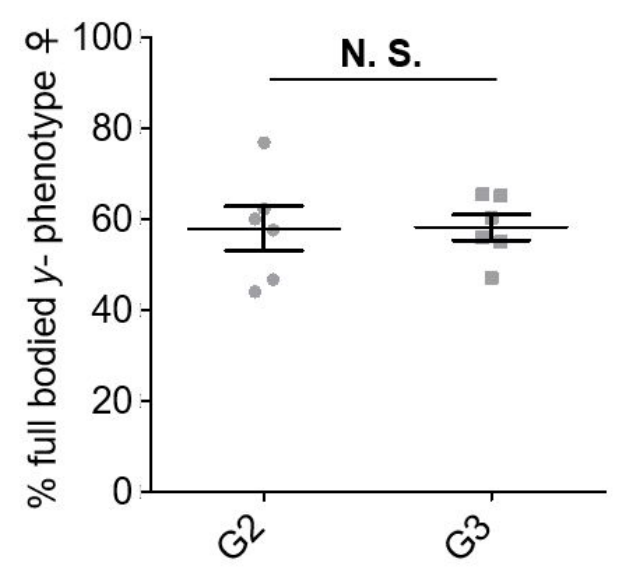

C

\begin{tabular}{c|c|c} 
& $y$-female & wild-type female \\
\hline G1-2 & 127 & 61 \\
\hline G2-3 & 233 & $\begin{array}{l}127 \\
p=0.5701 \dagger\end{array}$
\end{tabular}

\section{G1 female}

Figure S3. Gene drive efficiency remains stable over generations. (A) Full-bodied y-phenotype $\mathrm{G}_{1}$ females were mated with wild-type males and the female progeny were analyzed to calculate the efficiency of gene drive. $G_{3}$ progenies were obtained by mating wild-type males with $G_{2}$ females. (B) Comparison of gene drive efficiencies over generations. $G_{1}-G_{2}$ and $G_{2}-G_{3}$ refer to full-bodied $y$ phenotype $G_{2}$ female progeny proportions and the percentage of full-bodied y- phenotype $G_{3}$ females among the total number of $G_{3}$ females, with the exception of those showing a mosaic phenotype, derived from a single full-bodied $y$ - phenotype $G_{1}$ female, respectively. $n$ (number of $G_{1} y$ - females) $=6$ for $F_{1}-F_{2}$ and 6 for $F_{2}-F_{3}$. Error bars represent the s.e.m. NS, no statistical significance, Student's t-test. (C) Pearson's chi-squared test performed on the contingency table of $y$ - and wild-type females across the G1-2 and G2-3. 
A

wild-type $\sigma^{x}$ x G1 full bodied $y$ - phenotype 우

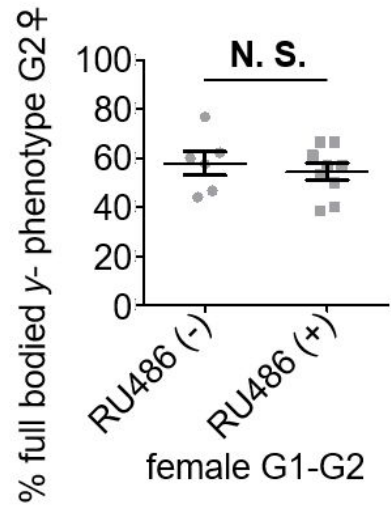

B

G1 full bodied $y$ - phenotype $\sigma^{7} \mathrm{x}$ wild-type 우

N.S.

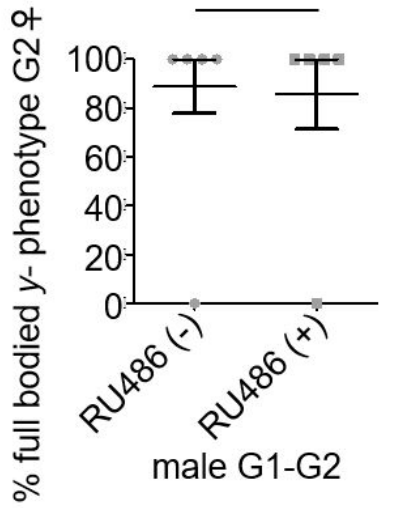

Figure S4. Phenotypes of $\mathrm{G} 2$ females when the $y-\mathrm{G} 1$ flies were crossed with wild type flies in the presence of RU486. (A) Full-bodied $y$ - phenotype $G_{2}$ female progeny proportions derived from a cross between a single full-bodied $y$ - phenotype $\mathrm{G}_{1}$ female and a single $w^{1118}$ male with or without $200 \mu \mathrm{M}$ RU486. $\mathrm{n}$ (number of $\mathrm{G}_{1} \mathrm{y}^{-}$females) $=6$ for $0 \mu \mathrm{M}$ RU486 and 9 for $200 \mu \mathrm{M}$ RU486. Error bars represent the s.e.m. Student's t-test NS, no statistical significance. (B) Full-bodied $y$-phenotype $\mathrm{G}_{2}$ female progeny proportions derived from a cross between a single full-bodied $y$ - phenotype $G_{1}$ male and a single $w^{1118}$ female with or without $200 \mu M$ RU486. $n$ (number of $G_{1} y^{-}$females) $=9$ for $0 \mu M$ RU486 and 7 for $200 \mu \mathrm{M}$ RU486. Error bars represent the s.e.m. Student's t-test. NS, no statistical significance. 


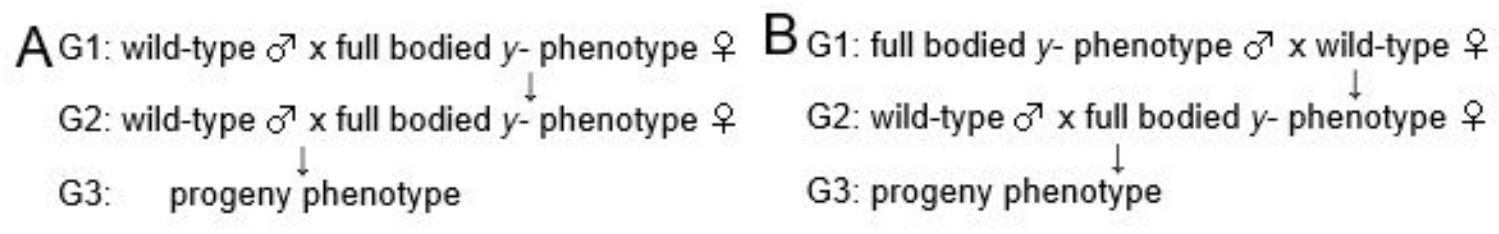

C

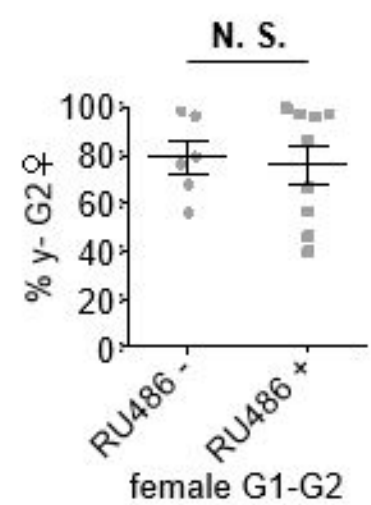

E

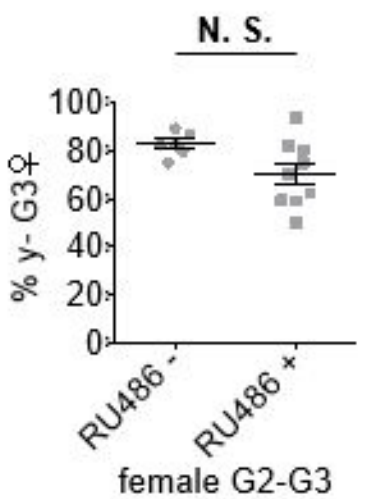

$\mathrm{D}$

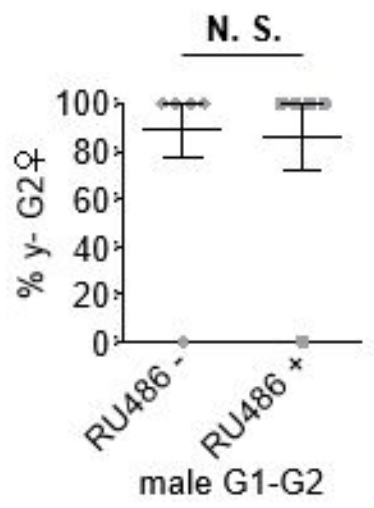

$\mathrm{F}$

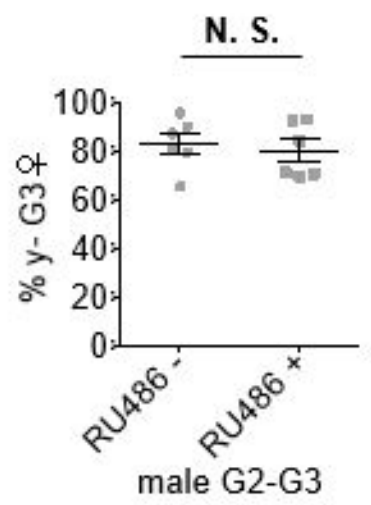

Figure S5. Phenotypic analysis of the chemically induced turning off of gene drive when mosaic $y$ phenotype females are counted. (A and $\mathbf{B}$ ) Schematics of individual experiments in which the gene drive cassette was removed by administrating RU486 3-5 days before and during crossing. All $G_{2}$ offspring derived from crosses between a full-bodied $y$ - phenotype male and a wild-type female and between a full-bodied y- phenotype female and a wild-type male were counted. Representative fullbodied y- phenotype $G_{2}$ female progeny were crossed with wild-type males and the y phenotype of all offspring was evaluated. (C) $y-G_{2}$ female progeny proportions derived from crosses between fullbodied y- phenotype $G_{1}$ female parents with or without $200 \mu \mathrm{M}$ RU486 and wild-type males. $\mathrm{n}$ (number of $G_{1} y$ - males) $=6$ for $0 \mu \mathrm{M}$ RU486 and 9 for $200 \mu \mathrm{M}$ RU486. Error bars represent the s.e.m. NS, no statistical significance, Student's t-test. (D) $y-G_{2}$ female progeny proportions derived from crosses between full-bodied $y$ - phenotype $G_{1}$ male parents with or without $200 \mu M$ RU486 and wild-type females. $\mathrm{n}$ (number of $\mathrm{G}_{1} \mathrm{y}$ - females) $=9$ for $0 \mu \mathrm{M}$ RU486 and 7 for $200 \mu \mathrm{M}$ RU486. Error bars represent the s.e.m. NS, no statistical significance, Student's t-test. (E) $y-G_{3}$ female progeny proportions derived from a cross between a single full-bodied $y$ - phenotype $G_{2}$ female and a single $w^{1118}$ male with or without $200 \mu M$ RU486. Each dot represents the percentage of $y-G_{3}$ females among the total number of $G_{3}$ females derived from a single full-bodied y- phenotype $G_{1}$ female. $n$ (number of $\mathrm{G}_{1} \mathrm{y}$ - males) $=6$ for $0 \mu \mathrm{M}$ RU486 and 9 for $200 \mu \mathrm{M}$ RU486. Error bars represent the s.e.m. NS, no statistical significance, Student's t-test. $(\mathbf{F}) y-G_{3}$ female progeny proportions derived from a cross between a single full-bodied y- phenotype $G_{2}$ female and a single $w^{1118}$ male with or without $200 \mu \mathrm{M}$ RU486. Each dot represents the percentage of $y-G_{3}$ females among the total number of $G_{3}$ females derived from a single full-bodied $y$ - phenotype $G_{1}$ male. $n$ (number of $G_{1} y$ - males) $=6$ for $0 \mu \mathrm{M}$ RU486 and 6 for $200 \mu \mathrm{M}$ RU486. Error bars represent the s.e.m. NS, no statistical significance, Student's t-test. 


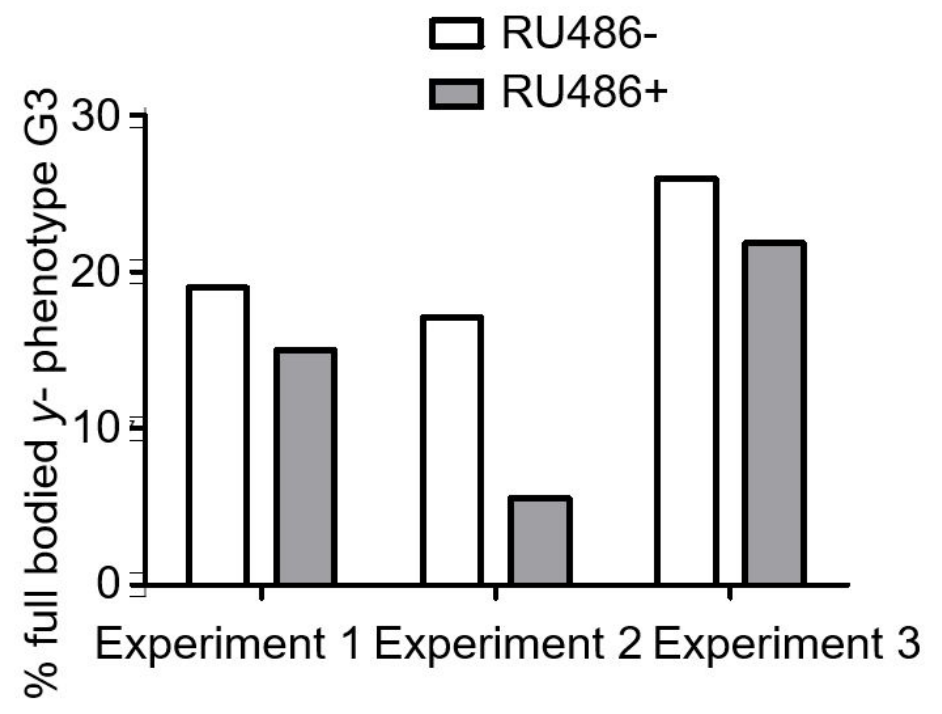

Figure S6. Effect of RU486 on the percentage of full-bodied y-phenotype individuals in populationbased cage experiments. 


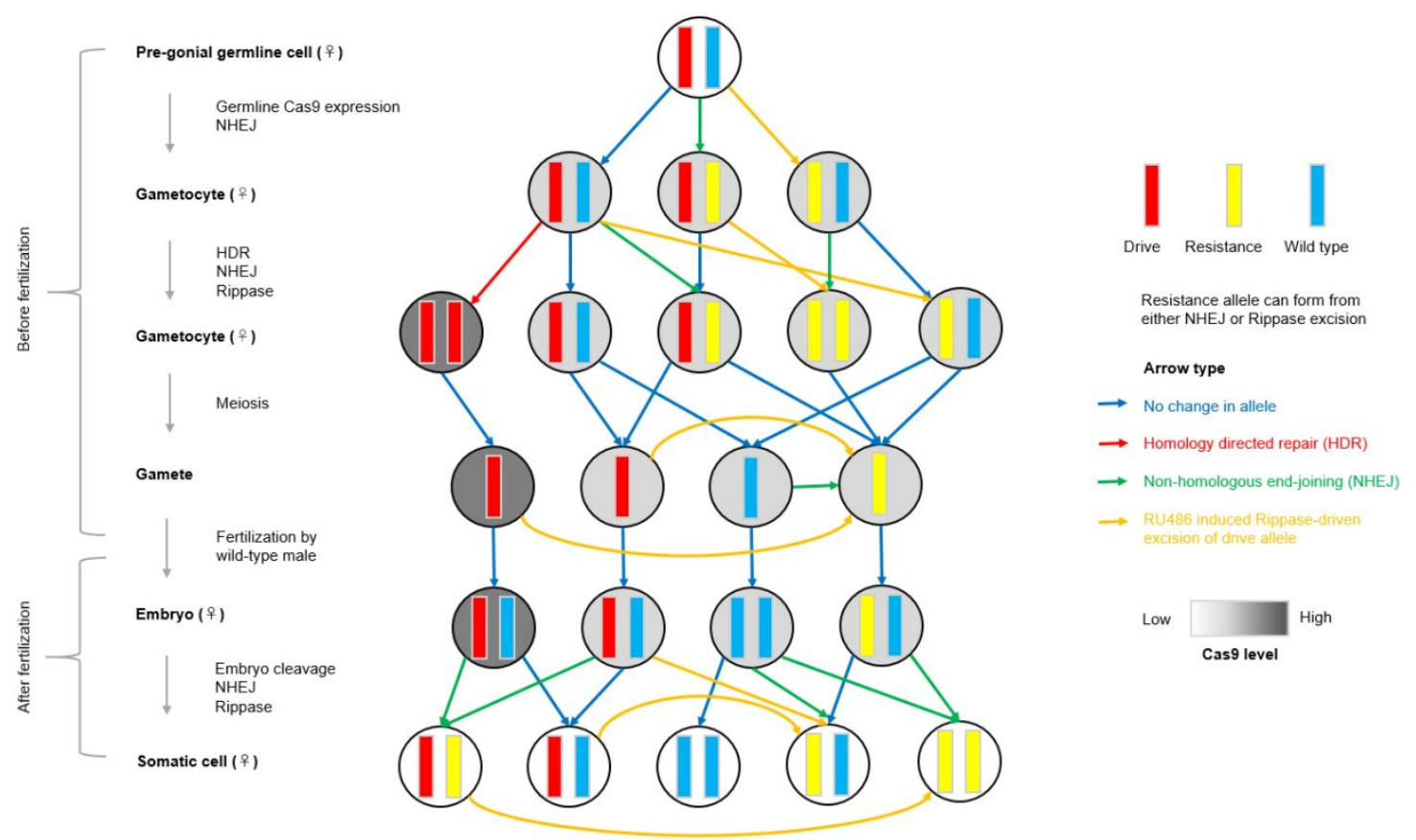

Figure S7. Mechanisms of RU486 action. In a female with genotype D/+, resistance alleles can form before fertilization either through RU486 induced Rippase-driven excision or NHEJ. After fertilization, Cas9 persisting in the cytoplasm of females with genotypes $\mathrm{D} /+,+/+$, and $\mathrm{r} 2 /+$ can convert the wildtype allele to r2. RU486 induced Rippase-driven excision in females with genotypes D/+ and D/D (resulting from homing in late embryogenesis or after birth; not shown in the figure), converts the wildtype allele to $r$. 
A

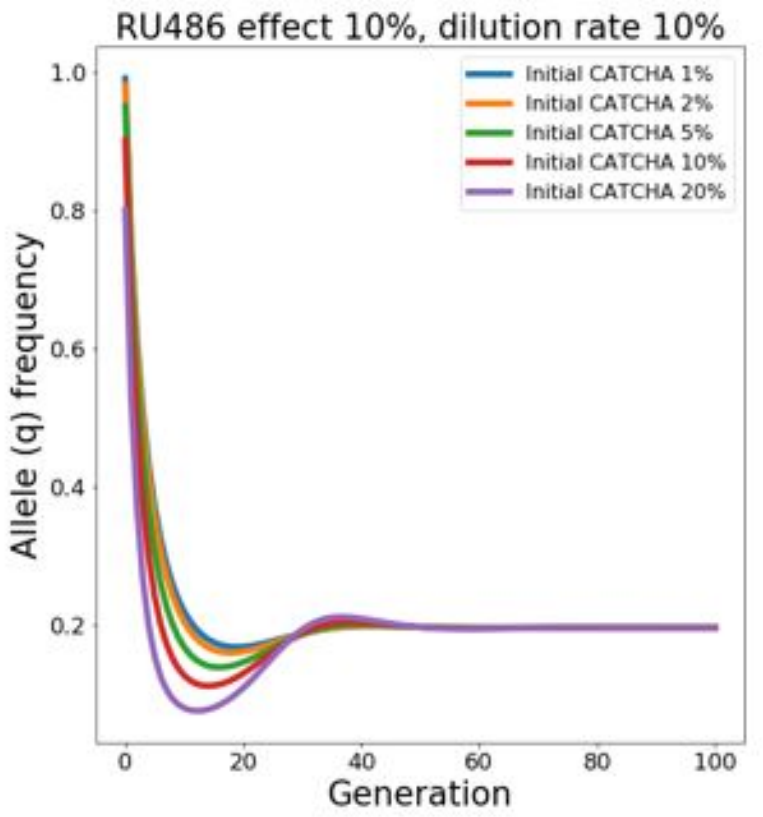

C

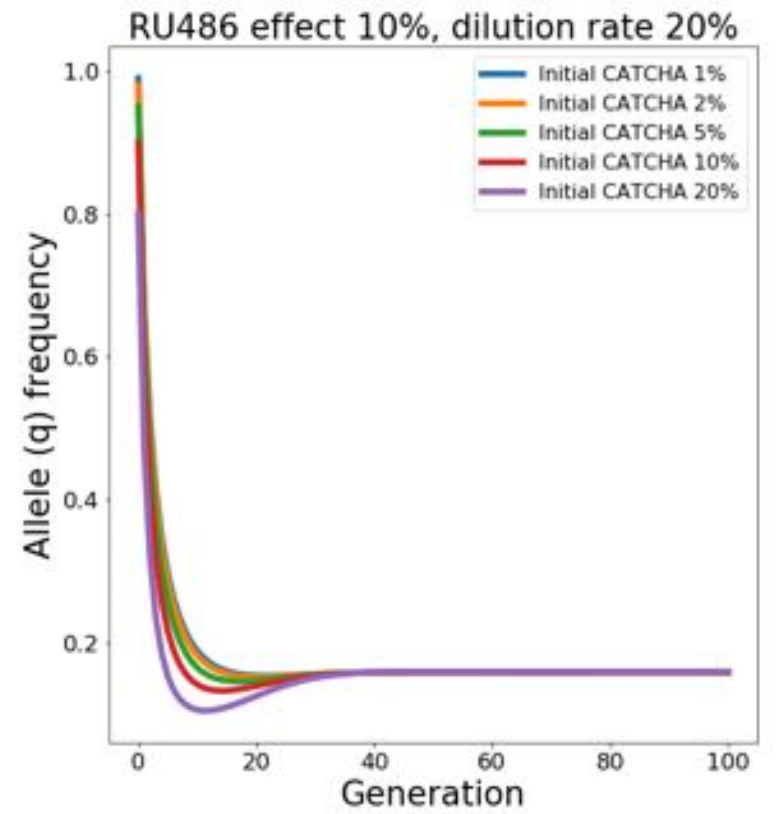

B

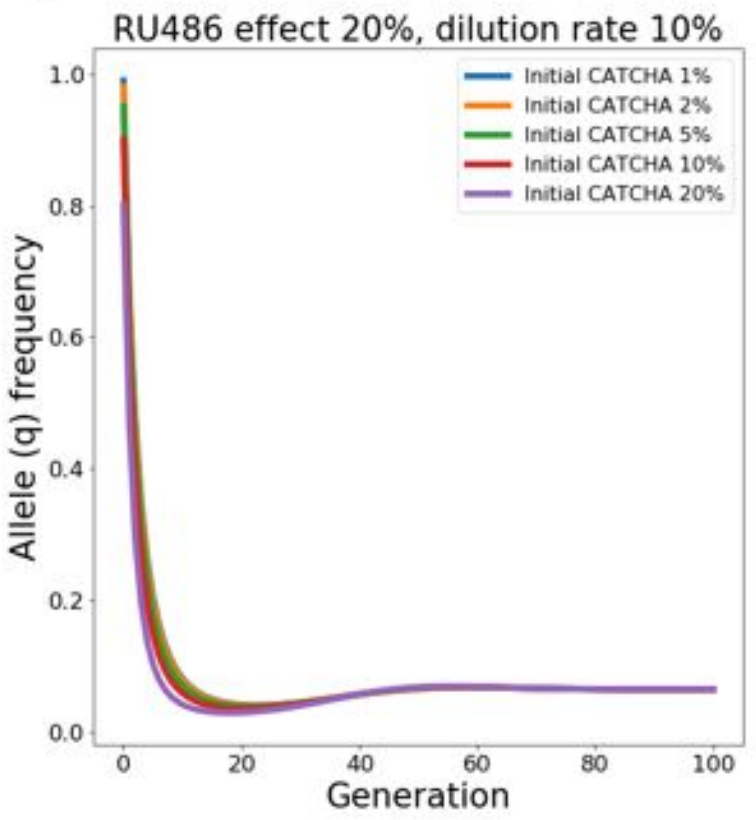

D

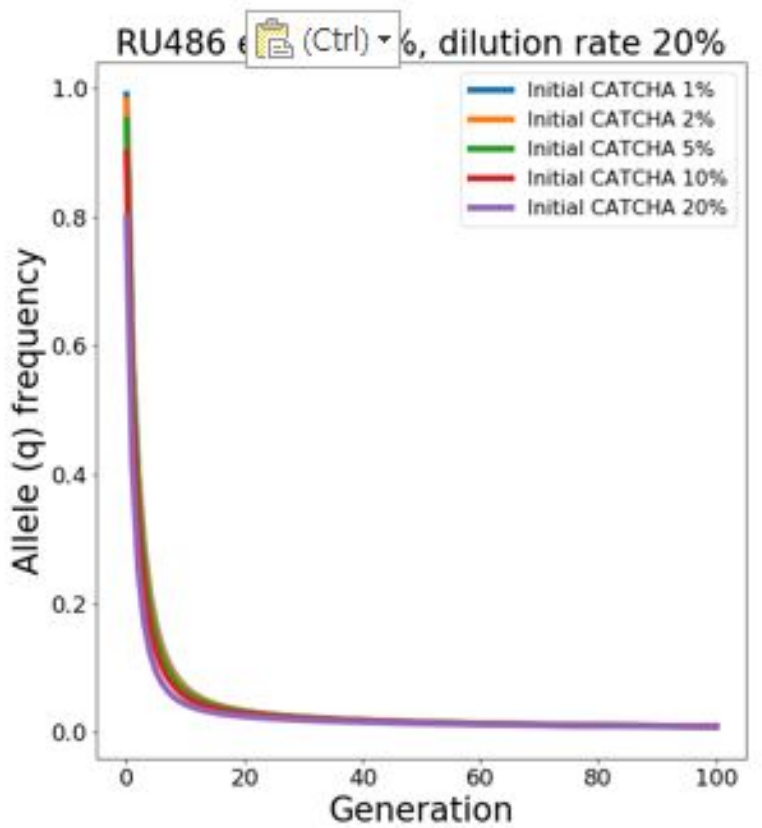

Figure S8. Simulations of combining CATCHA with CGD, assuming a range of different initial CATCHA allele frequency (s), RU486 effects, and dilution rates. (A) RU486 effect $10 \%$ and dilution rate $10 \%$, (B) RU486 effect $20 \%$ and dilution rate $10 \%$, (C) RU486 effect $10 \%$ and dilution rate $20 \%$, (D) RU486 effect $20 \%$ and dilution rate $20 \%$. Under WT immigration, co-treatment with CGD helps overcome CATCHA's limitation in achieving complete gene reversal. 
(A) CATCHA allele frequency (s)
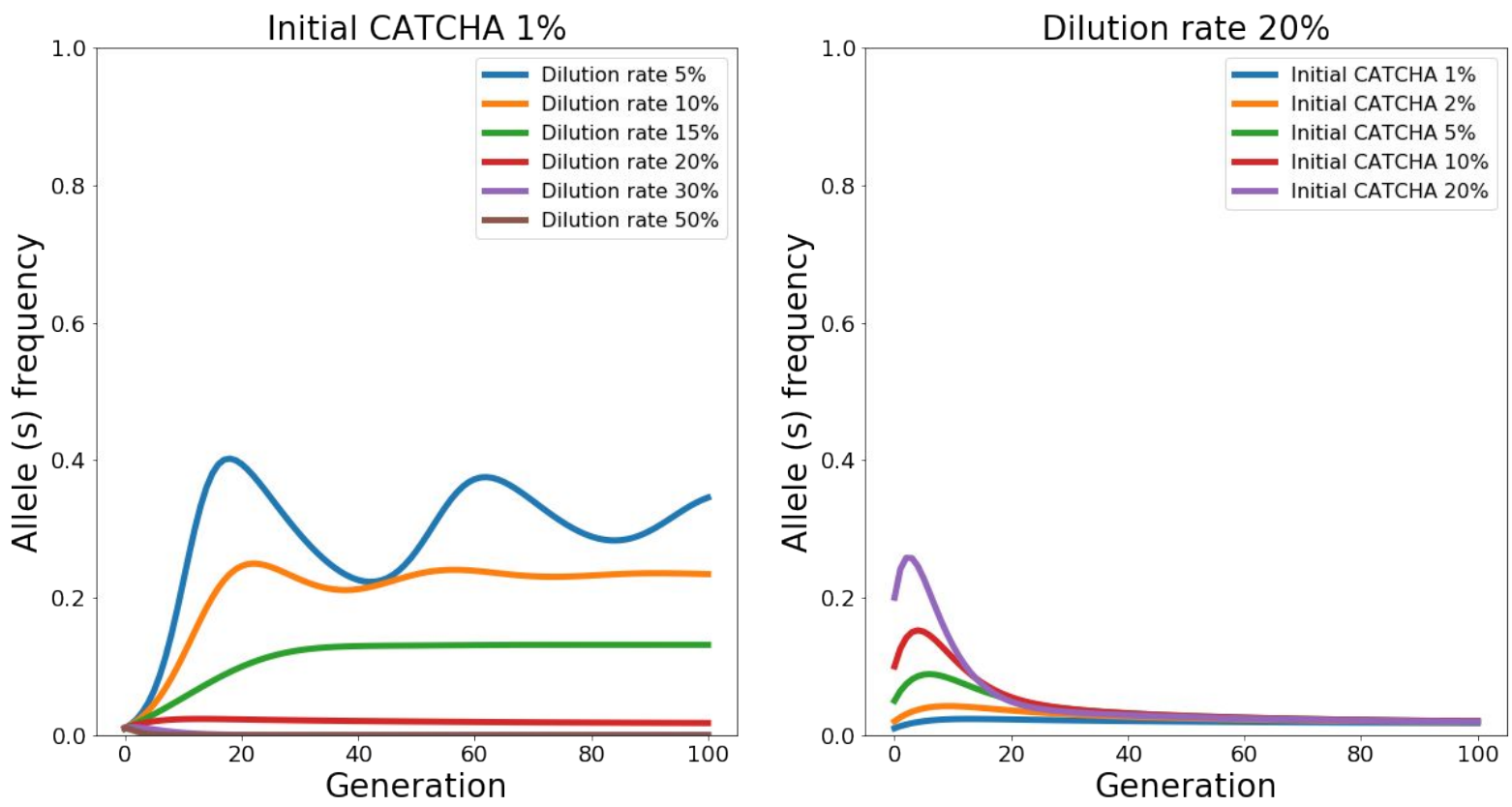

(B) $r 2$ allele frequency $(r)$
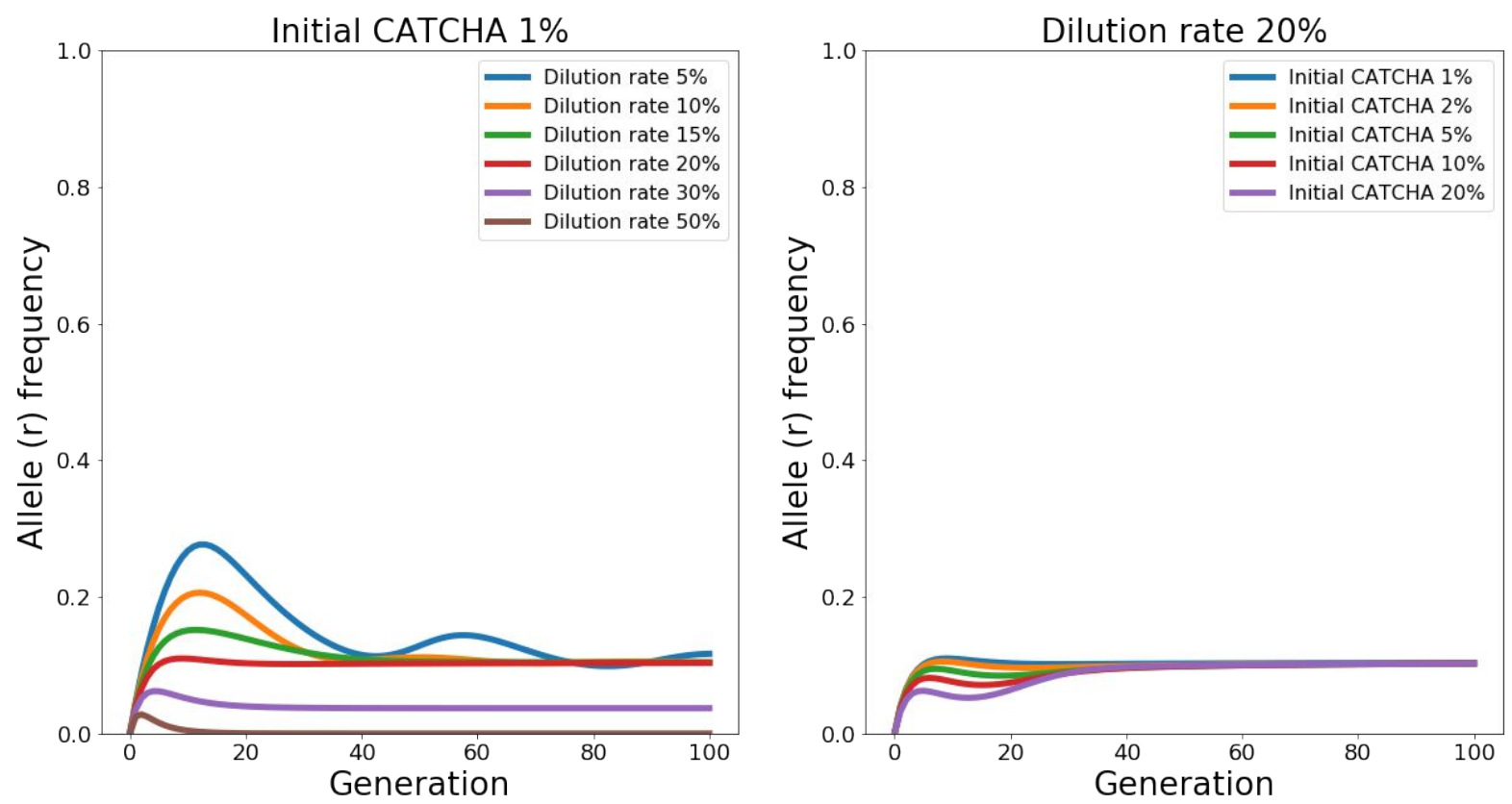

Figure S9. Open system simulation based on the same scenarios as in Figure 6. (A) CATCHA allele frequency, (B) $r 2$ allele frequency $(r)$. Dilution rate of $20 \%$ leads to an extinction of CATCHA that cannot be overcome by initial s as high as $20 \%$. 
(A) $\mathrm{NHEJ}=0 \%$

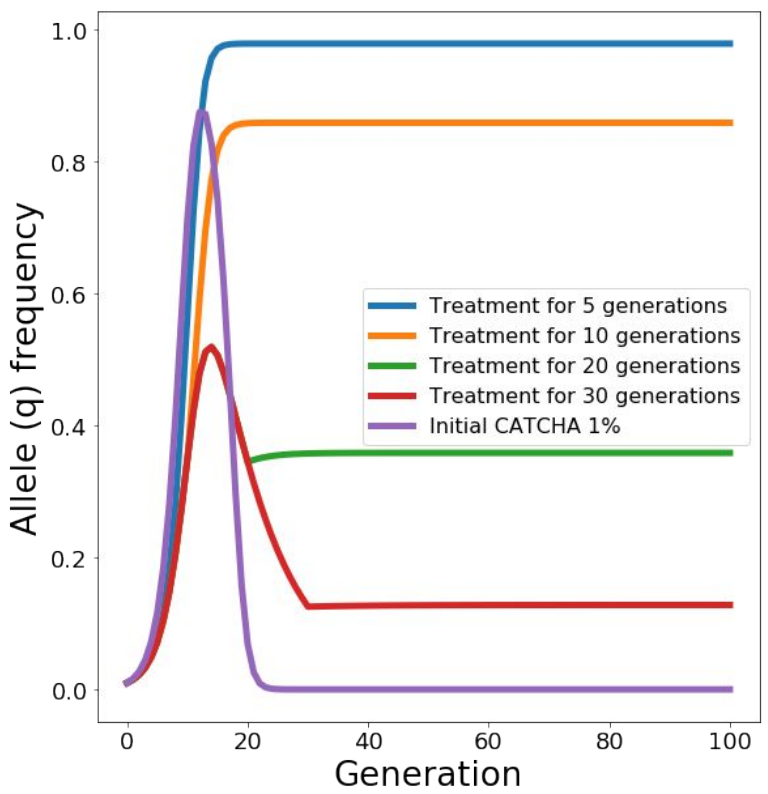

(B) $\mathrm{NHEJ}=5 \%$

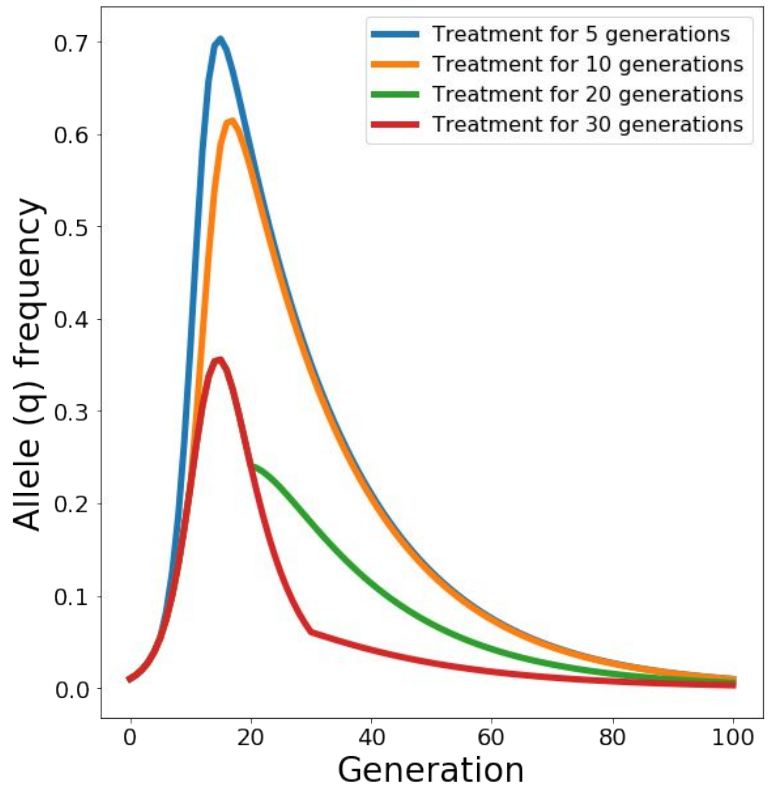

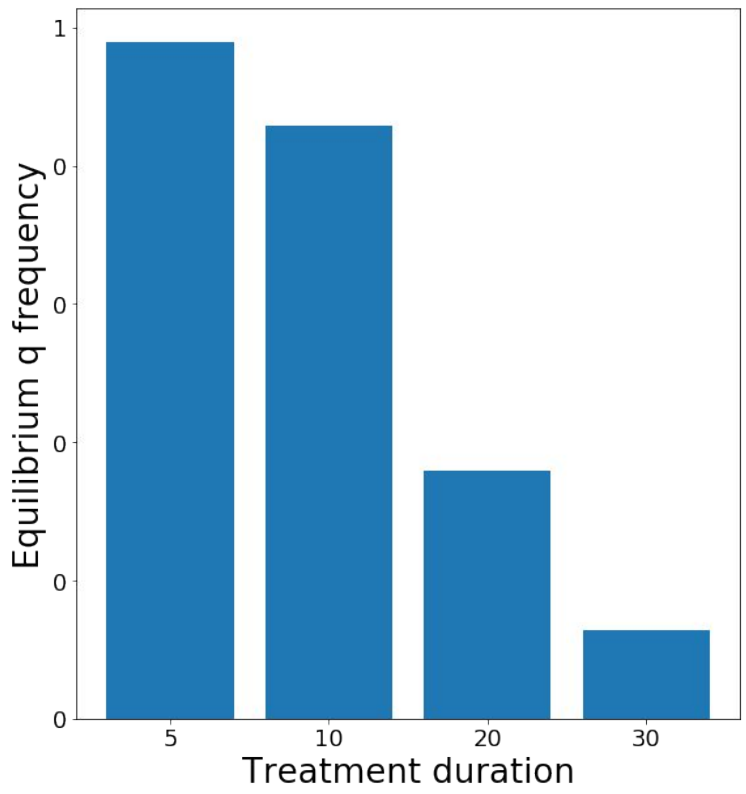

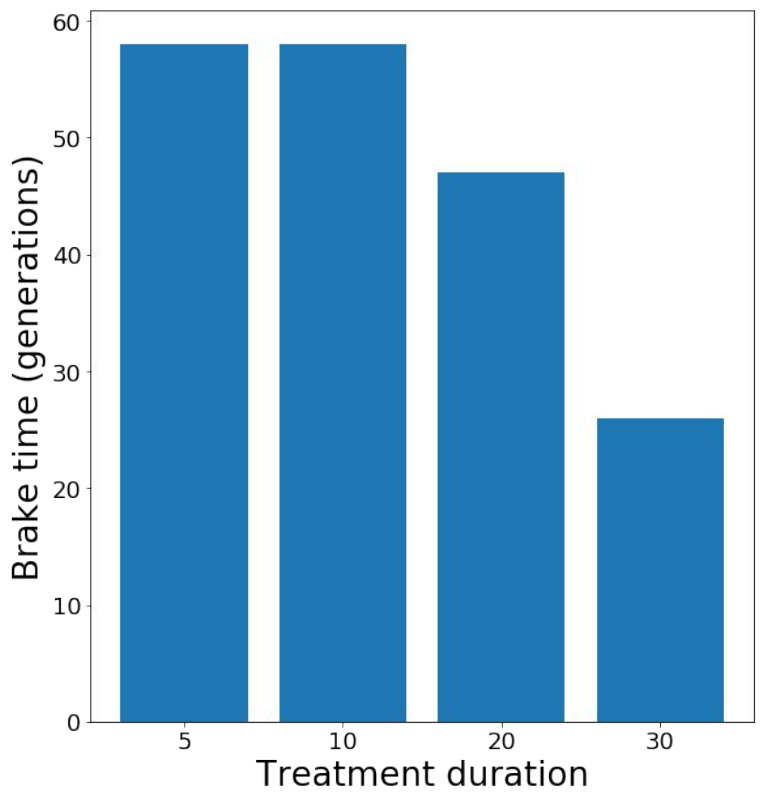

Figure S10. Reversibility of RU486 treatment. (A) RU486, as opposed to CATCHA, ceases to exert its effect the moment the treatment is withdrawn if resistance allele formation due to NHEJ is negligible. Simulations were performed assuming a 10\% RU486 effect and an initial CATCHA allele frequency of $1 \%$. Times of treatment withdrawal were set as the $5^{\text {th }}$ (blue), $10^{\text {th }}$ (orange), $20^{\text {th }}$ (green), and $30^{\text {th }}$ (red) generations. The effect of CATCHA cannot be undone once it is inoculated into the population. (B) When NHEJ occurs at a rate of $5 \%$, gene drive always comes to a halt. Different treatment durations lead to different brake times. 
Table S1. The sequences of primers used in this study.

\begin{tabular}{|l|l|l|l|}
\hline \multirow{2}{*}{$\begin{array}{l}\text { Out-in junction } \\
\text { PCR }\end{array}$} & Out-in F & F & 5'-GTTGCGAGGTTTTAGGACTGAAAGAGC-3' \\
\cline { 2 - 4 } & Out-in R & R & 5'-CCAGCTGGCGTAATAGCGAAGA-3' \\
\hline \multirow{2}{*}{$\begin{array}{l}\text { In-out junction } \\
\text { PCR }\end{array}$} & In-out F & F & 5'-TGGGGACCACTTGTCTCGTA-3' \\
\cline { 2 - 4 } & In-out R & R & 5'-CCTCCCCCTGAACCTGAAA-3' \\
\hline \multirow{2}{*}{ Absence PCR } & Y anterior & F & 5'-CATTAGCACGGCAGTTACCA-3' \\
\cline { 2 - 4 } & Y posterior & R & 5'-CCGAGAATCTCCAGGACTTG-3' \\
\hline
\end{tabular}

\begin{tabular}{|l|l|l|l|}
\hline \multirow{2}{*}{$\begin{array}{l}\text { Homology arm 1 } \\
\text { subcloning }\end{array}$} & $\begin{array}{l}\text { Homology } \\
\text { arm 1 }\end{array}$ & F & 5'- GATTGAAAGGTGCATTATGC -3' \\
\cline { 2 - 4 } & $\begin{array}{l}\text { Homology } \\
\text { arm 1 }\end{array}$ & R & 5'- TTCCAGTGTCCAAAACCCAC -3' \\
\hline \multirow{2}{*}{$\begin{array}{l}\text { Homology arm 2 } \\
\text { subcloning }\end{array}$} & $\begin{array}{l}\text { Homology } \\
\text { arm 2 }\end{array}$ & F & 5'- CCGTGGGCATCGGCAATA -3' \\
\cline { 2 - 4 } & $\begin{array}{l}\text { Homology } \\
\text { arm 2 }\end{array}$ & R & 5'- CTGGAGACTACATTGCCTG -3' \\
\hline $\begin{array}{l}\text { Guide RNA } \\
\text { subcloning to } \\
\text { pCFD3- } \\
\text { dU6:3gRNA } \\
\text { (Addgene \# } \\
\text { 49410) }\end{array}$ & $\begin{array}{l}\text { Annealing } \\
\text { primer of y1 } \\
\text { gRNA }\end{array}$ & F & 5'- GTCGGTTTTGGACACTGGAACCG -3' \\
\cline { 2 - 4 } & $\begin{array}{l}\text { Annealing } \\
\text { primer of y1 } \\
\text { gRNA }\end{array}$ & R & 5'- AAACCGGTTCCAGTGTCCAAAAC -3' \\
\hline
\end{tabular}


Table S2. Summary of $G_{2}$ progeny obtained from gene drive crosses described in detail in Supplementary Tables S3-S12

\begin{tabular}{|c|c|c|c|c|c|c|}
\hline & $y-\hat{0}$ & $\mathrm{y}-9$ & Mosaic $\rho$ & $y+\delta^{\lambda}$ & $y+q$ & total \\
\hline full-boc & 0 & 179 & 0 & 208 & 25 & 412 \\
\hline full-bodied y- phenotype & 263 & 200 & 73 & 34 & 61 & 631 \\
\hline
\end{tabular}


Table S3. $G_{2}$ progenies obtained by crossing full-bodied y- phenotype $G_{1}$ males with wild-type females in the absence of RU486

\begin{tabular}{|c|c|c|c|c|c|c|c|c|c|c|c|}
\hline $\begin{array}{l}\mathrm{G}_{0} \\
\text { progenitor }\end{array}$ & $\begin{array}{l}\text { Name } \\
\text { of } G_{1}\end{array}$ & $\begin{array}{l}\text { Total number } \\
\text { of } \\
\text { offspring }\end{array}$ & $\begin{array}{l}G_{2} \\
y- \\
\lambda^{2}\end{array}$ & $\begin{array}{l}G_{2} \\
y^{+} \\
\lambda^{+}\end{array}$ & $\begin{array}{l}G_{2} \\
y- \\
0\end{array}$ & $\begin{array}{l}G_{2} \\
y^{+} \\
0\end{array}$ & $\begin{array}{l}\text { Mosaic } \\
0 \\
+\end{array}$ & $\begin{array}{l}\text { Total } \\
\text { number } \\
\mathrm{G}_{2} \text { O }\end{array}$ & $\begin{array}{l}\text { Total } \\
\text { of } \mid \begin{array}{l}\text { number } \\
\text { HDR }^{1} \text { O }\end{array}\end{array}$ & of $\mid \begin{array}{l}\% \text { full-bodied } y- \\
\text { phenotype } \mathrm{G}_{2} \mathrm{O}\end{array}$ & $\mid \begin{array}{ll}\% & y- \\
\mathrm{G}_{2}+\end{array}$ \\
\hline$y-\mathrm{G} 0-1$ & $0-1 M$ & 7 & 0 & 4 & 3 & 0 & 0 & 3 & 3 & 100 & 100 \\
\hline$y-G 0-1$ & $0-3 M$ & 56 & 0 & 38 & 18 & 0 & 0 & 18 & 18 & 100 & 100 \\
\hline$y-\mathrm{G} 0-1$ & $0-4 M$ & 53 & 0 & 28 & 0 & 25 & 0 & 25 & 0 & 0 & 0 \\
\hline$y-\mathrm{G} 0-1$ & $0-5 M$ & 34 & 0 & 19 & 15 & 0 & 0 & 15 & 15 & 100 & 100 \\
\hline$y-\mathrm{G} 0-2$ & $0-6 \mathrm{M}$ & 19 & 0 & 3 & 16 & 0 & 0 & 16 & 16 & 100 & 100 \\
\hline$y-\mathrm{G} 0-2$ & 0-7M & 9 & 0 & 4 & 5 & 0 & 0 & 5 & 5 & 100 & 100 \\
\hline$y-\mathrm{G} 0-2$ & 0-8M & 56 & 0 & 26 & 30 & 0 & 0 & 30 & 30 & 100 & 100 \\
\hline$y-\mathrm{G} 0-2$ & 0-9M & 86 & 0 & 43 & 43 & 0 & 0 & 43 & 43 & 100 & 100 \\
\hline$y-\mathrm{G} 0-2$ & 0-10M & 92 & 0 & 43 & 49 & 0 & 0 & 49 & 49 & 100 & 100 \\
\hline
\end{tabular}

${ }^{1}$ homology-directed repair 
Table S4. $G_{2}$ progenies obtained by crossing full-bodied y- phenotype $G_{1}$ males with wild-type females in the presence of RU486

\begin{tabular}{|c|c|c|c|c|c|c|c|c|c|c|c|}
\hline $\begin{array}{l}\mathrm{G}_{0} \\
\text { progenitor }\end{array}$ & $\begin{array}{l}\text { Name } \\
\text { of } G_{1}\end{array}$ & $\begin{array}{l}\text { Total } \\
\text { number o } \\
\mathrm{G}_{2} \text { offspring }\end{array}$ & $\begin{array}{l}G_{2} \\
y- \\
0\end{array}$ & $\begin{array}{l}G_{2} \\
y+3\end{array} \mid$ & $\mid \begin{array}{l}G_{2} \\
y-q\end{array}$ & $\begin{array}{l}G_{2} \\
y+o\end{array}$ & $\begin{array}{l}\text { Mosaic } \\
+ \\
+\end{array}$ & $\mid \begin{array}{l}\text { Total } \\
\text { number o } \\
\mathrm{G}_{2}+\end{array}$ & $\begin{array}{l}\text { Total } \\
\text { number } \\
\text { HDR }^{1} \text { q }\end{array}$ & of $\mid \begin{array}{ll}\% & \text { full-bodied } \\
\mathrm{G}_{2} & \text { phenotype } \\
\mathrm{G}_{2}+\end{array}$ & $\begin{array}{cc}\% & y- \\
G_{2} & +\end{array}$ \\
\hline$y-\mathrm{G} 0-1$ & $\begin{array}{l}200- \\
2 \mathrm{M}\end{array}$ & 78 & 1 & 37 & 40 & 0 & 0 & 40 & 40 & 100 & 100 \\
\hline$y-\mathrm{G} 0-1$ & $\begin{array}{l}200- \\
4 \mathrm{M}\end{array}$ & 134 & 0 & 78 & 56 & 0 & 0 & 56 & 56 & 100 & 100 \\
\hline$y-\mathrm{G} 0-1$ & $\begin{array}{l}200- \\
5 \mathrm{M}\end{array}$ & 220 & 0 & 98 & 0 & 122 & 0 & 122 & 0 & 0 & 0 \\
\hline$y-\mathrm{G} 0-2$ & $\begin{array}{l}200- \\
6 \mathrm{M}\end{array}$ & 236 & 0 & 119 & 117 & 0 & 0 & 117 & 117 & 100 & 100 \\
\hline$y-\mathrm{G} 0-2$ & $\begin{array}{l}200- \\
7 \mathrm{M}\end{array}$ & 59 & 0 & 26 & 0 & 33 & 0 & 33 & 0 & 0 & 0 \\
\hline$y-\mathrm{G} 0-2$ & $\begin{array}{l}200- \\
8 \mathrm{M}\end{array}$ & 122 & 0 & 61 & 61 & 0 & 0 & 61 & 61 & 100 & 100 \\
\hline$y-\mathrm{G} 0-2$ & $\begin{array}{l}200- \\
9 M\end{array}$ & 86 & 0 & 39 & 47 & 0 & 0 & 47 & 47 & 100 & 100 \\
\hline$y-\mathrm{G} 0-2$ & $\begin{array}{l}200- \\
10 M\end{array}$ & 94 & 0 & 44 & 50 & 0 & 0 & 50 & 50 & 100 & 100 \\
\hline
\end{tabular}

${ }^{1}$ homology-directed repair 
Table S5. $G_{2}$ progenies obtained by crossing full-bodied $y$ - phenotype $G_{1}$ females with wild-type males in the absence of RU486

\begin{tabular}{|c|c|c|c|c|c|c|c|c|c|c|c|}
\hline $\begin{array}{l}\mathrm{G}_{0} \\
\text { progenitor }\end{array}$ & $\begin{array}{l}\text { Name } \\
\text { of } G_{1}\end{array}$ & $\begin{array}{l}\text { Total numb } \\
\text { of } \\
\text { offspring }\end{array}$ & $\mathrm{G}_{2} \mid \begin{array}{l}\mathrm{y}_{2} \\
\hat{\gamma}-\end{array}$ & $2 \begin{array}{l}G_{2} \\
y+ \\
\hat{\gamma}\end{array}$ & \begin{tabular}{|l|}
$\mathrm{G}_{2}$ \\
$\mathrm{y}-$ \\
+ \\
+
\end{tabular} & $\begin{array}{l}G_{2} \\
y+ \\
0\end{array}$ & $\begin{array}{l}\text { Mosaic } \\
0 \\
+\end{array}$ & $\begin{array}{l}\text { Total } \\
\text { number } \\
\mathrm{G}_{2}+\end{array}$ & $\begin{array}{l}\text { Total } \\
\text { of } \begin{array}{l}\text { number } \\
\mathrm{HDR}^{1} \text { Q }\end{array}\end{array}$ & of $\begin{array}{l}\% \text { full-bodied } y \\
\text { phenotype } G_{2}\end{array}$ & $\mathrm{G}_{2} \mathrm{y}$ \\
\hline$y-\mathrm{G} 0-1$ & $0-2 F$ & 183 & 76 & 9 & 61 & 20 & 17 & 98 & 78 & 62.24 & 79.59 \\
\hline$y-\mathrm{G} 0-1$ & $0-3 F$ & 102 & 41 & 2 & 34 & 2 & 23 & 59 & 57 & 57.63 & 96.61 \\
\hline$y-\mathrm{G} 0-1$ & $0-4 \mathrm{~F}$ & 140 & 71 & 0 & 53 & 1 & 15 & 69 & 68 & 76.81 & 98.55 \\
\hline$y-\mathrm{G} 0-1$ & $0-5 \mathrm{~F}$ & 57 & 22 & 5 & 14 & 7 & 9 & 30 & 23 & 46.67 & 76.67 \\
\hline$y-\mathrm{G} 0-2$ & $0-6 \mathrm{~F}$ & 90 & 31 & 9 & 22 & 22 & 6 & 50 & 28 & 44 & 56 \\
\hline$y-G 0-2$ & 0-7F & 56 & 22 & 9 & 15 & 8 & 2 & 25 & 17 & 60 & 68 \\
\hline$y-\mathrm{G} 0-2$ & $0-8 \mathrm{~F}$ & 3 & 0 & 0 & 1 & 1 & 1 & 3 & 2 & 33.33 & 66.67 \\
\hline
\end{tabular}

${ }^{1}$ homology-directed repair 
Table S6. $G_{2}$ progenies obtained by crossing full-bodied y- phenotype $G_{1}$ females with wild-type males in the presence of RU486

\begin{tabular}{|c|c|c|c|c|c|c|c|c|c|c|c|}
\hline $\begin{array}{l}\mathrm{G}_{0} \\
\text { progenitor }\end{array}$ & $\mid \begin{array}{l}\text { Name } \\
\text { of } \mathrm{G}_{1}\end{array}$ & $\begin{array}{l}\text { Total numb } \\
\text { of } \\
\text { offspring }\end{array}$ & $G_{2} \mid \begin{array}{l}G_{2} \\
y- \\
\hat{j}\end{array}$ & $\mid \begin{array}{l}G_{2} \\
y+ \\
y^{\lambda}\end{array}$ & $\begin{array}{l}G_{2} \\
y- \\
0 \\
+\end{array}$ & $\begin{array}{l}G_{2} \\
y+ \\
+9\end{array}$ & $\begin{array}{l}\text { Mosaic } \\
0 \\
+\end{array}$ & $\begin{array}{l}\text { Total } \\
\text { number } \\
G_{2}+\end{array}$ & of $\begin{array}{l}\text { Total } \\
\text { number } \\
\text { HDR }^{1} \text { o }\end{array}$ & of $\mid \begin{array}{l}\% \text { full-bodied } y- \\
\text { phenotype } G_{2}\end{array}$ & $\%_{0} y-$ \\
\hline$y-\mathrm{G} 0-1$ & 200-1F & $=65$ & 25 & 3 & 21 & 1 & 15 & 37 & 36 & 56.76 & 97.30 \\
\hline$y-\mathrm{G} 0-1$ & $200-2 F$ & $=78$ & 41 & 10 & 21 & 1 & 15 & 37 & 36 & 56.76 & 97.30 \\
\hline$y-\mathrm{G} 0-1$ & $200-3 F$ & $=52$ & 31 & 10 & 14 & 0 & 7 & 21 & 21 & 66.67 & 100 \\
\hline$y-\mathrm{G} 0-1$ & $200-4 \mathrm{~F}$ & $=85$ & 57 & 71 & 18 & 1 & 8 & 27 & 26 & 66.67 & 96.30 \\
\hline$y-\mathrm{G} 0-1$ & $200-5 F$ & $F 19$ & 6 & 3 & 4 & 6 & 0 & 10 & 4 & 40 & 40 \\
\hline$y-\mathrm{G} 0-2$ & $200-6 \mathrm{~F}$ & $=45$ & 17 & 72 & 10 & 14 & 2 & 26 & 12 & 38.46 & 46.15 \\
\hline$y-\mathrm{G} 0-2$ & 200-7F & $=105$ & 38 & \begin{tabular}{l|l}
8 & 13
\end{tabular} & 33 & 18 & 3 & 54 & 36 & 61.11 & 66.67 \\
\hline$y-\mathrm{G} 0-2$ & $200-8 \mathrm{~F}$ & $=69$ & 21 & 14 & 22 & 19 & 3 & 44 & 25 & 50 & 56.82 \\
\hline$y-\mathrm{G} 0-2$ & $200-9 F$ & $=28$ & 11 & 12 & 8 & 2 & 5 & 15 & 13 & 53.33 & 86.67 \\
\hline
\end{tabular}

${ }^{1}$ homology-directed repair 
Table S7. $G_{3}$ progenies obtained by crossing full-bodied y- phenotype $G_{2}$ females, derived from y- $G_{1}$ males in the absence of RU486, with wild-type males in the absence of RU486

\begin{tabular}{|c|c|c|c|c|c|c|c|c|c|c|c|c|c|}
\hline $\begin{array}{l}\text { Name } \\
\text { of } G_{1}\end{array}$ & $\begin{array}{l}\text { Name } \\
\text { of } G_{2}\end{array}$ & $\begin{array}{l}\text { Total } \\
\text { number o } \\
G_{3} \\
\text { offspring }\end{array}$ & $\mid \begin{array}{l}G_{3} \\
y- \\
\lambda^{\prime}\end{array}$ & $\begin{array}{l}\mathrm{G}_{3} \\
y^{+} \\
\hat{j}^{-}\end{array}$ & $\begin{array}{l}\mathrm{G}_{3} \\
y- \\
9 \\
+\end{array}$ & $\begin{array}{l}G_{3} \\
y+ \\
0 \\
+\end{array}$ & $\begin{array}{l}\text { Mosaic } \\
+\end{array}$ & 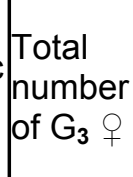 & $\begin{array}{l}\text { Total } \\
\text { number } \\
\text { of } \\
\text { ond }\end{array}$ & $\begin{array}{l}\% \\
\text { bodied full } y \\
\text { phenotype } \\
G_{3} \text { ? }\end{array}$ & $\begin{array}{ll}\% & y \\
\mathrm{G}_{3} & \end{array}$ & $\begin{array}{l}\% \\
\text { bodied } \\
\text { phenotype } \\
G_{3}+\end{array}$ & $\%_{G_{3}} \quad y-$ \\
\hline $0-3 M$ & $0-3-1$ & 55 & 23 & 1 & 22 & 1 & 8 & 31 & 30 & 70.97 & 96.77 & \multirow{5}{*}{66.89} & \multirow{5}{*}{90.18} \\
\hline $0-3 \mathrm{M}$ & $0-3-2$ & 81 & 44 & 3 & 24 & 3 & 7 & 34 & 31 & 70.59 & 91.18 & & \\
\hline $0-3 \mathrm{M}$ & $0-3-3$ & 70 & 37 & 1 & 20 & 4 & 8 & 32 & 28 & 62.5 & 87.5 & & \\
\hline $0-3 \mathrm{M}$ & $0-3-4$ & 83 & 36 & 8 & 23 & 4 & 12 & 39 & 35 & 58.97 & 89.74 & & \\
\hline $0-3 \mathrm{M}$ & $0-3-5$ & 13 & 4 & 2 & 5 & 1 & 1 & 7 & 6 & 71.43 & 85.71 & & \\
\hline $0-5 \mathrm{M}$ & $0-5-2$ & 65 & 32 & 3 & 17 & 3 & 10 & 30 & 27 & 56.67 & 90 & \multirow{4}{*}{64.83} & \multirow{4}{*}{$95.7 \varepsilon$} \\
\hline $0-5 \mathrm{M}$ & $0-5-3$ & 58 & 17 & 0 & 30 & 0 & 11 & 41 & 41 & 73.17 & 100 & & \\
\hline $0-5 \mathrm{M}$ & $0-5-4$ & 78 & 31 & 0 & 34 & 1 & 12 & 47 & 46 & 72.34 & 97.87 & & \\
\hline $0-5 \mathrm{M}$ & $0-5-5$ & 44 & 23 & 0 & 12 & 1 & 8 & 21 & 20 & 57.14 & 95.24 & & \\
\hline $0-6 \mathrm{M}$ & $0-6-1$ & 5 & 2 & 0 & 3 & 0 & 0 & 3 & 3 & 100 & 100 & \multirow{5}{*}{62.2} & \multirow{5}{*}{87.5} \\
\hline $0-6 \mathrm{M}$ & $0-6-2$ & 64 & 22 & 6 & 19 & 8 & 9 & 36 & 28 & 52.78 & 77.78 & & \\
\hline $0-6 \mathrm{M}$ & $0-6-3$ & 74 & 33 & 7 & 19 & 8 & 7 & 34 & 26 & 55.88 & 76.47 & & \\
\hline $0-6 \mathrm{M}$ & $0-6-4$ & 79 & 33 & 3 & 23 & 0 & 20 & 43 & 43 & 53.49 & 100 & & \\
\hline $0-6 \mathrm{M}$ & $0-6-5$ & 78 & 30 & 5 & 21 & 7 & 15 & 43 & 36 & 48.84 & 83.72 & & \\
\hline 0-8M & $0-8-1$ & 75 & 33 & 7 & 20 & 11 & 4 & 35 & 24 & 57.14 & 68.57 & \multirow{5}{*}{57.08} & \multirow{5}{*}{79.83} \\
\hline 0-8M & $0-8-2$ & 62 & 27 & 8 & 14 & 4 & 9 & 27 & 23 & 51.85 & 85.19 & & \\
\hline 0-8M & $0-8-3$ & 73 & 34 & 6 & 22 & 6 & 5 & 33 & 27 & 66.67 & 81.82 & & \\
\hline $0-8 \mathrm{M}$ & $0-8-4$ & 59 & 26 & 10 & 11. & 4 & 8 & 23 & 19 & 47.83 & 82.61 & & \\
\hline 0-8M & $0-8-5$ & 76 & 26 & 8 & 26 & 8 & 8 & 42 & 34 & 61.90 & 80.95 & & \\
\hline 0-9M & $0-9-1$ & 62 & 20 & 6 & 20 & 5 & 11 & 36 & 31 & 55.56 & 86.11 & \multirow{3}{*}{53.47} & \multirow{3}{*}{81.35} \\
\hline 0-9M & $0-9-2$ & 62 & 25 & 6 & 21 & 6 & 4 & 31 & 25 & 67.74 & 80.65 & & \\
\hline 0-9M & $0-9-3$ & 70 & 32 & 2 & 21 & 6 & 9 & 36 & 30 & 58.33 & 83.33 & & \\
\hline
\end{tabular}




\begin{tabular}{|c|c|c|c|c|c|c|c|c|c|c|c|c|c|}
\hline $0-9 M$ & 0-9-4 & 46 & 19 & & 9 & 2 & 9 & 20 & 18 & 45 & 90 & & \\
\hline $0-9 \mathrm{M}$ & $0-9-5$ & 60 & 23 & 10 & 11 & 9 & 7 & 27 & 18 & 40.74 & 66.67 & & \\
\hline 0-10M & $\mid 0-10-1$ & 83 & 32 & 12 & 23 & 8 & 8 & 39 & 31 & 58.97 & 79.49 & \multirow{5}{*}{53.23} & \multirow{5}{*}{65.71} \\
\hline $0-10 \mathrm{M}$ & $0-10-2$ & 21 & 13 & 4 & 2 & 2 & 0 & 4 & 2 & 50 & 50 & & \\
\hline $0-10 \mathrm{M}$ & $0-10-3$ & 88 & 34 & 16 & 20 & 9 & 9 & 38 & 29 & 52.63 & 76.32 & & \\
\hline $0-10 \mathrm{M}$ & $0-10-4$ & 2 & 0 & 0 & 1 & 1 & 0 & 2 & 1 & 50 & 50 & & \\
\hline $0-10 \mathrm{M}$ & $0-10-5$ & & 12 & & 6 & 3 & 2 & 11 & 8 & 54.55 & 72.73 & & \\
\hline
\end{tabular}

${ }^{1}$ homology-directed repair 
Table S8. $G_{3}$ progenies obtained by crossing full-bodied $y$ - phenotype $G_{2}$ females, derived from $y-G_{1}$ males in the presence of RU486, with wild-type males in the presence of RU486

\begin{tabular}{|c|c|c|c|c|c|c|c|c|c|c|c|c|c|}
\hline $\begin{array}{l}\text { Name } \\
\text { of } G_{1}\end{array}$ & $\begin{array}{l}\text { Name } \\
\text { of } G_{2}\end{array}$ & $\begin{array}{l}\text { Total } \\
\text { number o } \\
\mathrm{G}_{3} \\
\text { offspring }\end{array}$ & $\left|\begin{array}{c}G_{3} \\
y- \\
\lambda \\
\lambda\end{array}\right|$ & $\mid \begin{array}{c}\mathrm{G}_{3} \\
\mathrm{y} \\
\hat{0}\end{array}$ & \begin{tabular}{l|l}
$\mathrm{G}_{3}$ & $\mathrm{G}$ \\
$\mathrm{y}-$ & $\mathrm{y}$ \\
& \\
+ & $\mathrm{O}$
\end{tabular} & $\begin{array}{c}\mathrm{G}_{3} \\
\mathrm{y}^{+} \\
x^{2}\end{array}$ & $\begin{array}{l}\text { Mosaic } \\
q\end{array}$ & $\left\{\begin{array}{l}\text { Total } \\
\text { number } \\
\text { of } \mathrm{G}_{3} \text { o }\end{array}\right.$ & $\begin{array}{l}\text { Total } \\
\text { number } \\
\text { of } \\
\text { of }\end{array}$ & $\begin{array}{lr}\% & \text { full } \\
\text { bodied } y \\
\text { phenotype } \\
G_{3}+\end{array}$ & $\begin{array}{ll}\% & y- \\
\mathrm{G}_{3} & \end{array}$ & $\begin{array}{l}\% \\
\text { bodied full } y \\
\text { phenotype } \\
G_{3}+\end{array}$ & $\begin{array}{ll}\% & y- \\
\mathrm{G}_{3} & \end{array}$ \\
\hline $\begin{array}{l}200- \\
2 M\end{array}$ & $\begin{array}{l}200-2- \\
1\end{array}$ & 59 & 31 & & $16 \mid 0$ & \begin{tabular}{l|l}
0 & 1
\end{tabular} & 11 & 27 & 27 & 59.26 & 100 & \multirow{5}{*}{53.27} & \multirow{5}{*}{93.19} \\
\hline \begin{tabular}{|l}
$200-$ \\
$2 M$
\end{tabular} & $\begin{array}{l}200-2- \\
2\end{array}$ & 60 & 255 & 5 & 152 & \begin{tabular}{l|l}
2 & 1
\end{tabular} & 13 & 30 & 28 & 50 & 93.33 & & \\
\hline \begin{tabular}{|l}
$200-$ \\
$2 \mathrm{M}$
\end{tabular} & $\begin{array}{l}200-2- \\
3\end{array}$ & 60 & 276 & 6 & 142 & \begin{tabular}{l|l}
2 & 1
\end{tabular} & 11 & 27 & 25 & 51.85 & 92.59 & & \\
\hline $\begin{array}{l}200- \\
2 M\end{array}$ & $\begin{array}{l}200-2- \\
4\end{array}$ & 73 & $31<$ & & 215 & $5 \quad 1$ & 12 & 38 & 33 & 55.26 & 86.84 & & \\
\hline $\begin{array}{l}200- \\
2 \mathrm{M}\end{array}$ & $\begin{array}{l}200-2- \\
5\end{array}$ & 65 & $\left.18\right|^{3}$ & 3 & 223 & \begin{tabular}{l|l}
3 & 1
\end{tabular} & 19 & 44 & 41 & 50 & 93.18 & & \\
\hline $\begin{array}{l}200- \\
4 \mathrm{M}\end{array}$ & $\begin{array}{l}200-4- \\
1\end{array}$ & 42 & 16 & 0 & 150 & & 11 & 26 & 26 & 57.69 & 100 & & \\
\hline $\begin{array}{l}200- \\
4 \mathrm{M}\end{array}$ & $\begin{array}{l}200-4- \\
2\end{array}$ & 36 & 20 & 1 & $\begin{array}{ll}8 & 1\end{array}$ & 16 & 6 & 15 & 14 & 53.33 & 93.33 & & \\
\hline $\begin{array}{l}200- \\
4 \mathrm{M}\end{array}$ & $\begin{array}{l}200-4- \\
3\end{array}$ & 58 & $\left.30\right|_{2} ^{2}$ & 2 & 142 & $2 \quad 1$ & 10 & 26 & 24 & 53.85 & 92.31 & 60.49 & 93.39 \\
\hline $\begin{array}{l}200- \\
4 \mathrm{M}\end{array}$ & $\begin{array}{l}200-4- \\
4\end{array}$ & 69 & 18 & 1 & 345 & $5 \quad 1$ & 11 & 50 & 45 & 68 & 90 & & \\
\hline $\begin{array}{l}200- \\
4 \mathrm{M}\end{array}$ & $\begin{array}{l}200-4- \\
5\end{array}$ & 45 & 20 & 2 & $16 \mid 2$ & 25 & 5 & 23 & 21 & 69.57 & 91.30 & & \\
\hline $\begin{array}{l}200- \\
6 \mathrm{M}\end{array}$ & $\begin{array}{l}200-6- \\
1\end{array}$ & 46 & $17 \mid 2$ & 2 & 155 & $5 \quad 7$ & 7 & 27 & 22 & 55.56 & 81.48 & & \\
\hline $\begin{array}{l}200- \\
6 \mathrm{M}\end{array}$ & $\begin{array}{l}200-6- \\
2\end{array}$ & 41 & $19 \epsilon^{6}$ & 6 & \begin{tabular}{|l|l}
8 & 5
\end{tabular} & 53 & 3 & 16 & 11 & 50 & 68.75 & & \\
\hline $\begin{array}{l}200- \\
6 \mathrm{M}\end{array}$ & $\begin{array}{l}200-6- \\
3\end{array}$ & 23 & 5 & 5 & $\begin{array}{ll}7 & 4\end{array}$ & 42 & 2 & 13 & 9 & 53.85 & 69.23 & 50.75 & 70.89| \\
\hline $\begin{array}{l}200- \\
6 \mathrm{M}\end{array}$ & $\begin{array}{l}200-6-6 \\
4\end{array}$ & 45 & 14 & 6 & \begin{tabular}{l|l}
9 & 1
\end{tabular} & 106 & 6 & 25 & 15 & 36 & 60 & & \\
\hline $\begin{array}{l}200- \\
6 \mathrm{M}\end{array}$ & $\begin{array}{l}200-6- \\
5\end{array}$ & 54 & 20 & 10 & $14 \mid 6$ & & 4 & 24 & 18 & 58.33 & 75 & & \\
\hline $\begin{array}{l}200- \\
8 \mathrm{M}\end{array}$ & $\begin{array}{l}200-8- \\
1\end{array}$ & 54 & 225 & 5 & 146 & & 7 & 27 & 21 & 51.85 & 77.78 & & \\
\hline \begin{tabular}{|l|}
$200-$ \\
$8 \mathrm{M}$
\end{tabular} & $\begin{array}{l}200-8- \\
2\end{array}$ & 36 & 112 & & 137 & & 3 & 23 & 16 & 56.52 & 69.57 & .15 & 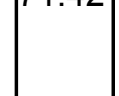 \\
\hline
\end{tabular}




\begin{tabular}{|c|c|c|c|c|c|c|c|c|c|c|c|c|c|}
\hline $\begin{array}{l}200- \\
8 \mathrm{M}\end{array}$ & $\begin{array}{l}200-8- \\
3\end{array}$ & 88 & 31 & 11 & \begin{tabular}{|l|l}
19 & 1
\end{tabular} & $19 \mid \varepsilon$ & 8 & 46 & 27 & 41.30 & 58.70 & & \\
\hline $\begin{array}{l}200- \\
8 \mathrm{M}\end{array}$ & $\begin{array}{l}200-8- \\
4\end{array}$ & 83 & 30 & 16 & $20 \mid 1$ & $11 k$ & 6 & 37 & 26 & 54.05 & 70.27 & & \\
\hline $\begin{array}{l}200- \\
8 \mathrm{M}\end{array}$ & $\begin{array}{l}200-8 \\
5\end{array}$ & 50 & $|17| 7$ & 7 & 135 & 5 & 8 & 26 & 21 & 50 & 80.77 & & \\
\hline $\begin{array}{l}200- \\
9 \mathrm{M}\end{array}$ & $\begin{array}{l}200-9 \\
1\end{array}$ & 62 & 223 & 3 & $|19| 8$ & 8 & 10 & 37 & 29 & 51.35 & 78.38 & \multirow{5}{*}{52.54} & \multirow{5}{*}{84.48} \\
\hline $\begin{array}{l}200- \\
9 \mathrm{M}\end{array}$ & $\begin{array}{l}200-9- \\
2\end{array}$ & 69 & $\left.24\right|_{2} ^{2}$ & 2 & $26 \mid 3$ & 3 & 14 & 43 & 40 & 60.47 & 93.02 & & \\
\hline $\begin{array}{l}200- \\
9 M\end{array}$ & $\begin{array}{l}200-9- \\
3\end{array}$ & 63 & $26 \mid$ & 4 & $15 \mid 2$ & 2 & 16 & 33 & 31 & 45.45 & 93.94 & & \\
\hline $\begin{array}{l}200- \\
9 \mathrm{M}\end{array}$ & $\begin{array}{l}200-9- \\
4\end{array}$ & 54 & 23 & 1 & \begin{tabular}{|l|l}
111 & 1
\end{tabular} & $11 \varepsilon$ & 8 & 30 & 19 & 36.67 & 63.33 & & \\
\hline $\begin{array}{l}200- \\
9 \mathrm{M}\end{array}$ & $\begin{array}{l}200-9- \\
5\end{array}$ & 28 & 11 & 1 & \begin{tabular}{|l|l|}
11 & 1
\end{tabular} & 1 & 4 & 16 & 15 & 68.75 & 93.75 & & \\
\hline $\begin{array}{l}200- \\
10 \mathrm{M}\end{array}$ & $\begin{array}{l}200- \\
10-1\end{array}$ & 17 & $10 \mid$ & 1 & $\begin{array}{ll}3 & 2\end{array}$ & 2 & 1 & 6 & 4 & 50 & 66.67 & \multirow{5}{*}{49.82} & \multirow{5}{*}{69.89} \\
\hline $\begin{array}{l}200- \\
10 M\end{array}$ & $\begin{array}{l}200- \\
10-2\end{array}$ & 58 & $\left.21\right|_{2} ^{2}$ & 2 & \begin{tabular}{|l|l|}
17 & 1
\end{tabular} & $10 \mid \varepsilon$ & 8 & 35 & 25 & 48.57 & 71.43 & & \\
\hline $\begin{array}{l}200- \\
10 M\end{array}$ & $\begin{array}{l}200- \\
10-3\end{array}$ & 19 & 5 & 2 & \begin{tabular}{|l|l}
7 & 4
\end{tabular} & 4 & 1 & 12 & 8 & 58.33 & 66.67 & & \\
\hline $\begin{array}{l}200- \\
10 M\end{array}$ & $\begin{array}{l}200- \\
10-4\end{array}$ & 53 & $21 s$ & 9 & $|10| 8$ & 8 & 5 & 23 & 15 & 43.48 & 65.22 & & \\
\hline $\begin{array}{l}200- \\
10 M\end{array}$ & $\begin{array}{l}200- \\
10-5\end{array}$ & 73 & 24 & 10 & 198 & 8 & 12 & 39 & 31 & 48.72 & 79.49 & & \\
\hline
\end{tabular}

${ }^{1}$ homology-directed repair 
Table S9. $G_{3}$ progenies obtained by crossing full-bodied $y$ - phenotype $G_{2}$ females, derived from $y-G_{1}$ females in the absence of RU486, with wild-type males in the absence of RU486

\begin{tabular}{|c|c|c|c|c|c|c|c|c|c|c|c|c|c|}
\hline $\begin{array}{l}\text { Name } \\
\text { of } G_{1}\end{array}$ & $\begin{array}{l}\text { Name } \\
\text { of } G_{2}\end{array}$ & $\begin{array}{l}\text { Total } \\
\text { number of } \\
G_{3} \\
\text { offspring }\end{array}$ & $\mid \begin{array}{l}G_{3} \\
y- \\
0 \\
\lambda\end{array}$ & $\begin{array}{c}G_{3} \\
y+ \\
\lambda\end{array}$ & 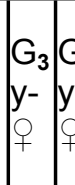 & $\begin{array}{c}\mathrm{G}_{3} \\
\mathrm{y}^{+} \\
+\end{array}$ & $\begin{array}{l}\text { Mosaic } \\
+ \\
+\end{array}$ & $\begin{array}{l}\text { Total } \\
\text { number } \\
\text { of } G_{3}+\end{array}$ & $\begin{array}{l}\text { Total } \\
\text { number } \\
\text { of HDR }{ }^{1} \\
\text { or }\end{array}$ & 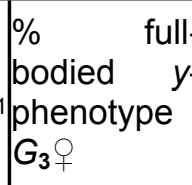 & $\begin{array}{ll}\% & y \\
G_{3} & \end{array}$ & $\begin{array}{l}\% \\
\text { bodied full } y \\
\text { phenotype } \\
G_{3} \subsetneq\end{array}$ & $\%$ \\
\hline $0-2 F$ & $0-2-1$ & 57 & 262 & 2 & 172 & 2 & 10 & 29 & 27 & 58.62 & 93.10 & \multirow{5}{*}{60.31} & \multirow{5}{*}{87.11} \\
\hline $0-2 F$ & $0-2-2$ & 46 & $14 \mid 7$ & 7 & $10 \mid 1$ & 114 & 4 & 25 & 14 & 40 & 56 & & \\
\hline $0-2 F$ & $0-2-3$ & 56 & 250 & 0 & $21 \mid 1$ & 16 & 9 & 31 & 30 & 67.74 & 96.77 & & \\
\hline $0-2 F$ & $0-2-4$ & 52 & $27 \mid c$ & 0 & $20 \mid 0$ & 05 & 5 & 25 & 25 & 80 & 100 & & \\
\hline $0-2 \mathrm{~F}$ & $0-2-5$ & 59 & 264 & 4 & 163 & 3 & 10 & 29 & 26 & 55.17 & 89.66 & & \\
\hline $0-3 F$ & $0-3-1$ & 70 & $30 \mid 8$ & 8 & $17 \mid 1$ & 150 & 0 & 32 & 17 & 53.13 & 53.13 & \multirow{5}{*}{47.1} & \multirow{5}{*}{82.46} \\
\hline $0-3 F$ & $0-3-2$ & 55 & 250 & 0 & 162 & & 12 & 30 & 28 & 53.33 & 93.33 & & \\
\hline $0-3 F$ & $0-3-3$ & 42 & 200 & 0 & 95 & 58 & 8 & 22 & 17 & 40.91 & 77.27 & & \\
\hline $0-3 F$ & $0-3-4$ & 51 & 26 & 0 & $|10| 1$ & 1 & 14 & 25 & 24 & 40 & 96 & & \\
\hline $0-3 F$ & $0-3-5$ & 52 & 232 & 2 & 132 & & 12 & 27 & 25 & 48.15 & 92.59 & & \\
\hline $0-4 \mathrm{~F}$ & 0-4-1 & 42 & 103 & 3 & 131 & 133 & 3 & 29 & 16 & 44.83 & 55.17 & \multirow{5}{*}{56.04} & \multirow{5}{*}{74.89} \\
\hline $0-4 \mathrm{~F}$ & $0-4-2$ & 47 & 230 & 0 & 160 & $\begin{array}{ll}0 & 8\end{array}$ & 8 & 24 & 24 & 66.67 & 100 & & \\
\hline $0-4 \mathrm{~F}$ & $0-4-3 \mid$ & 48 & $17 \mid c$ & 0 & 232 & 26 & 6 & 31 & 29 & 74.19 & 93.55 & & \\
\hline $0-4 \mathrm{~F}$ & $0-4-4$ & 40 & $24 \mid c$ & 0 & $|10| 1$ & 15 & 5 & 16 & 15 & 62.5 & 93.75 & & \\
\hline $0-4 \mathrm{~F}$ & $0-4-5$ & 45 & $|18|_{2}$ & 2 & \begin{tabular}{l|l}
8 & 1
\end{tabular} & $17 \mid c$ & 0 & 25 & 8 & 32 & 32 & & \\
\hline $0-5 F$ & $0-5-1$ & 73 & 283 & 3 & 241 & 108 & 8 & 42 & 32 & 57.14 & 76.19 & \multirow{3}{*}{55.01} & \multirow{3}{*}{79.78} \\
\hline $0-5 F$ & $0-5-2$ & 68 & 228 & 8 & 171 & 138 & 8 & 38 & 25 & 44.74 & 65.79 & & \\
\hline $5 \mathrm{~F}$ & $0-5-3$ & 61 & 230 & 0 & 241 & 1 & 13 & 38 & 37 & 63.16 & 97.37 & & \\
\hline
\end{tabular}




\begin{tabular}{|c|c|c|c|c|c|c|c|c|c|c|c|c|c|}
\hline $0-6 \mathrm{~F}$ & $0-6-1$ & 63 & $\left.18\right|^{7}$ & & \begin{tabular}{|l|l|l|}
22 & 8
\end{tabular} & $3 \varepsilon$ & 8 & 38 & 30 & 57.89 & 78.95 & \multirow{2}{*}{65.43} & \multirow{2}{*}{89.47} \\
\hline $0-6 F$ & $0-6-2$ & 65 & 280 & ) & 270 & & 10 & 37 & 37 & 72.97 & 100 & & \\
\hline $0-7 \mathrm{~F}$ & $0-7-1$ & 60 & 194 & & \begin{tabular}{|l|l}
23 & 4
\end{tabular} & & 10 & 37 & 33 & 62.16 & 89.19 & \multirow{3}{*}{65.13} & \multirow{3}{*}{82.57} \\
\hline $0-7 F$ & $0-7-2$ & 72 & 244 & & \begin{tabular}{|l|l}
27 & 1
\end{tabular} & 107 & 7 & 44 & 34 & 61.36 & 77.27 & & \\
\hline 0-7F & $0-7-3$ & 55 & $18 \mid 5$ & & 236 & & 3 & 32 & 26 & 71.88 & 81.25 & & \\
\hline
\end{tabular}

${ }^{1}$ homology-directed repair 
Table S10. $G_{3}$ progenies obtained by crossing full-bodied y- phenotype $G_{3}$ females, derived from y$\mathrm{G}_{1}$ females in the presence of RU486, with wild-type males in the presence of RU486

\begin{tabular}{|c|c|c|c|c|c|c|c|c|c|c|c|c|c|}
\hline $\begin{array}{l}\text { Name } \\
\text { of } G_{1}\end{array}$ & $\begin{array}{l}\text { Name } \\
\text { of } \mathrm{G}_{2}\end{array}$ & $\begin{array}{l}\text { Total } \\
\text { number o } \\
G_{3} \\
\text { offspring }\end{array}$ & $\mid \begin{array}{c}G_{3} \\
y- \\
0\end{array}$ & $\begin{array}{c}\mathrm{G}_{3} \\
\mathrm{y} \\
0^{\lambda}\end{array}$ & 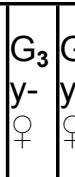 & $\begin{array}{l}\mathrm{G}_{3} \\
\mathrm{y}^{+} \\
\mathrm{O}^{+}\end{array}$ & $\begin{array}{l}\text { Mosaic } \\
0 \\
+\end{array}$ & $\begin{array}{l}\text { Total } \\
\text { number } \\
\text { of } G_{3} \text { 古 }\end{array}$ & $\begin{array}{ll}\text { Total } \\
\text { number } \\
\text { of } & \text { HDR }^{1} \\
\text { q } & \end{array}$ & $\begin{array}{l}\% \\
\text { bodied ful } \\
\text { phenotype } \\
G_{3} \text {, }\end{array}$ & $\begin{array}{ll}\% & y- \\
G_{3} & \end{array}$ & $\begin{array}{lr}\% & \text { full- } \\
\text { bodied } & y- \\
\text { phenotype } \\
G_{3}+\end{array}$ & $\begin{array}{ll}\% & y- \\
\mathrm{G}_{3} & \end{array}$ \\
\hline $\begin{array}{l}200- \\
1 \mathrm{~F}\end{array}$ & $\begin{array}{l}200-1- \\
2\end{array}$ & 52 & 29 & & 180 & \begin{tabular}{l|l}
0 & 5
\end{tabular} & 5 & 23 & 23 & 78.26 & 100 & \multirow{3}{*}{65.28} & \multirow{3}{*}{93.75} \\
\hline $\begin{array}{l}200- \\
1 \mathrm{~F}\end{array}$ & $\begin{array}{l}200-1- \\
3\end{array}$ & 63 & 25 & & 252 & \begin{tabular}{l|l}
2 & 1
\end{tabular} & 10 & 37 & 35 & 67.57 & 94.59 & & \\
\hline $\begin{array}{l}200- \\
1 \mathrm{~F}\end{array}$ & $\begin{array}{l}200-1- \\
5\end{array}$ & 63 & 33 & & $|15| 4$ & \begin{tabular}{l|l}
4 & 1
\end{tabular} & 11 & 30 & 26 & 50 & 86.67 & & \\
\hline $\begin{array}{l}200- \\
2 \mathrm{~F}\end{array}$ & $\begin{array}{l}200-2- \\
2\end{array}$ & 49 & 180 & & $\begin{array}{ll}0 & 3\end{array}$ & 310 & 0 & 31 & 0 & 0 & 0 & \multirow{2}{*}{16.67} & \multirow{2}{*}{50} \\
\hline $\begin{array}{l}200- \\
2 F\end{array}$ & $\begin{array}{l}200-2- \\
3\end{array}$ & 10 & 40 & 0 & 20 & 04 & 4 & 6 & 6 & 33.33 & 100 & & \\
\hline $\begin{array}{l}200- \\
3 \mathrm{~F}\end{array}$ & $\begin{array}{l}200-3-3 \\
1\end{array}$ & 44 & 220 & & \begin{tabular}{|l|l}
7 & 1
\end{tabular} & \begin{tabular}{l|l|}
1 & 1
\end{tabular} & 14 & 22 & 21 & 31.82 & 95.45 & \multirow{3}{*}{26.55} & \multirow{3}{*}{62.25} \\
\hline $\begin{array}{l}200- \\
3 F\end{array}$ & $\begin{array}{l}200-3- \\
2\end{array}$ & 48 & 250 & & 112 & & 10 & 23 & 21 & 47.83 & 91.30 & & \\
\hline $\begin{array}{l}200- \\
3 \mathrm{~F}\end{array}$ & \begin{tabular}{|l|}
$200-3-$ \\
3
\end{tabular} & 57 & 250 & & $\begin{array}{ll}0 & 3\end{array}$ & 320 & 0 & 32 & 0 & 0 & 0 & & \\
\hline $\begin{array}{l}200- \\
4 \mathrm{~F}\end{array}$ & $\begin{array}{l}200-4- \\
1\end{array}$ & 53 & $29 \mid 1$ & & $14 \mid 2$ & \begin{tabular}{l|l}
2 & 7
\end{tabular} & 7 & 23 & 21 & 60.87 & 91.30 & \multirow{3}{*}{39.99} & \multirow{3}{*}{59.22} \\
\hline $\begin{array}{l}200- \\
4 \mathrm{~F}\end{array}$ & $\begin{array}{l}200-4- \\
2\end{array}$ & 44 & $|19| 0$ & & $\begin{array}{ll}0 & 2\end{array}$ & 250 & 0 & 25 & 0 & 0 & 0 & & \\
\hline $\begin{array}{l}200- \\
4 \mathrm{~F}\end{array}$ & $\begin{array}{l}200-4- \\
4\end{array}$ & 51 & 272 & & $13 \mid 3$ & 36 & 6 & 22 & 19 & 59.09 & 86.36 & & \\
\hline $\begin{array}{l}200- \\
5 \mathrm{~F}\end{array}$ & $\begin{array}{l}200-5- \\
1\end{array}$ & 58 & 233 & & \begin{tabular}{|l|l}
14 & 1
\end{tabular} & 135 & 5 & 32 & 19 & 43.75 & 59.38 & 43.75 & 59.38 \\
\hline $\begin{array}{l}200- \\
6 \mathrm{~F}\end{array}$ & $\begin{array}{l}200-6- \\
1\end{array}$ & 57 & 252 & & \begin{tabular}{|l|l|}
13 & 1 \\
\end{tabular} & 125 & 5 & 30 & 18 & 43.33 & 60 & \multirow{3}{*}{48.61} & \multirow{3}{*}{70.5} \\
\hline $\begin{array}{l}200- \\
6 \mathrm{~F}\end{array}$ & $\begin{array}{l}200-6- \\
2\end{array}$ & 49 & 213 & & $10 \mid 9$ & 96 & 6 & 25 & 16 & 40 & 64 & & \\
\hline $\begin{array}{l}200- \\
6 \mathrm{~F}\end{array}$ & $\begin{array}{l}200-6- \\
3\end{array}$ & 71 & $\begin{array}{lll}36 & 3\end{array}$ & & 204 & $4 \quad 8$ & 8 & 32 & 28 & 62.5 & 87.5 & & \\
\hline $\begin{array}{l}200- \\
7 F\end{array}$ & $\begin{array}{l}200-7- \\
1\end{array}$ & 62 & 164 & & 247 & $7 \quad 1$ & 11 & 42 & 35 & 57.14 & 83.33 & \multirow{3}{*}{50.93} & \multirow{3}{*}{75.02} \\
\hline $\begin{array}{l}200- \\
7 \mathrm{~F}\end{array}$ & $\begin{array}{l}200-7- \\
2\end{array}$ & 54 & 154 & & 198 & \begin{tabular}{l|l}
8 & 8
\end{tabular} & 8 & 35 & 27 & 54.29 & 77.14 & & \\
\hline $\begin{array}{l}200- \\
7 F\end{array}$ & $\begin{array}{l}200-7- \\
3\end{array}$ & 61 & 225 & & 159 & & 10 & 34 & 25 & 44.12 & 73.53 & & \\
\hline
\end{tabular}




\begin{tabular}{|l|l|l|l|l|l|l|l|l|l|l|l|l|l|}
\hline $\begin{array}{l}200- \\
7 \mathrm{~F}\end{array}$ & $\begin{array}{l}200-7 \\
4\end{array}$ & 53 & 20 & 5 & 12 & 6 & 10 & 28 & 22 & 42.86 & 78.57 & & \\
\hline $\begin{array}{l}200- \\
7 \mathrm{~F}\end{array}$ \\
5
\end{tabular}

${ }^{1}$ homology-directed repair 
Table S11. $G_{3}$ progenies obtained by crossing mosaic $G_{2}$ females, derived from $y-G_{1}$ females in the absence of RU486, with wild-type males in the absence of RU486

\begin{tabular}{|c|c|c|c|c|c|c|c|c|c|c|c|}
\hline $\begin{array}{l}\text { Name } \\
\text { of } G_{1}\end{array}$ & $\begin{array}{l}\text { Name } \\
\text { of } G_{2}\end{array}$ & $\left|\begin{array}{ll}\text { Total } & \text { number } \\
\text { of } & \\
\text { offspring } & G_{3}\end{array}\right|$ & $\begin{array}{l}G_{3} \\
y- \\
- \\
\lambda\end{array}$ & $\begin{array}{l}\mathrm{G}_{3} \\
y^{+} \\
\hat{\lambda}\end{array}$ & $\mid \begin{array}{l}\mathrm{G}_{3} \\
\mathrm{y}- \\
0 \\
+\end{array}$ & $\mid \begin{array}{l}\mathrm{G}_{3} \\
y^{+} \\
+\end{array}$ & $\begin{array}{l}\text { Mosaic } \\
0\end{array}$ & $\begin{array}{l}\text { Total } \\
\text { number } \\
\mathrm{G}_{\mathbf{3}} \text { 早 }\end{array}$ & of $\begin{array}{l}\text { Total } \\
\text { number } \\
\mathrm{HDR}^{1} \text { क }\end{array}$ & of $\left[\begin{array}{l}\% \text { full-bodied } y \text { - } \\
\text { phenotype } G_{3} \text { ㅇ }\end{array}\right.$ & $\mid \begin{array}{ll}\% & y- \\
\mathrm{G}_{3} \bigcirc & \end{array}$ \\
\hline $0-2 \mathrm{~F}$ & $0-2-6$ & 60 & 14 & 16 & 0 & 30 & 0 & 30 & 0 & 0 & 0 \\
\hline $0-2 \mathrm{~F}$ & $0-2-7$ & 86 & 33 & 11 & 0 & 42 & 0 & 42 & 0 & 0 & 0 \\
\hline $0-2 F$ & $0-2-8$ & 75 & 24 & 18 & 0 & 33 & 0 & 33 & 0 & 0 & 0 \\
\hline $0-3 F$ & $0-3-6$ & 55 & 20 & 1 & 19 & 2 & 13 & 34 & 32 & 55.88 & 94.12 \\
\hline $0-3 F$ & $0-3-7$ & 62 & 21 & 6 & 0 & 35 & 0 & 35 & 0 & 0 & 0 \\
\hline $0-3 F$ & $0-3-8$ & 58 & 16 & 13 & 0 & 29 & 0 & 29 & 0 & 0 & 0 \\
\hline $0-3 F$ & 0-3-9 & 44 & 21 & 0 & 17 & 0 & 6 & 23 & 23 & 73.91 & 100 \\
\hline $0-4 \mathrm{~F}$ & $0-4-6$ & 56 & 23 & 4 & 0 & 29 & 0 & 29 & 0 & 0 & 0 \\
\hline $0-4 \mathrm{~F}$ & $0-4-7$ & 54 & 20 & 8 & 0 & 26 & 0 & 26 & 0 & 0 & 0 \\
\hline $0-4 \mathrm{~F}$ & 0-4-8 & 36 & 15 & 4 & 0 & 17 & 0 & 17 & 0 & 0 & 0 \\
\hline $0-4 \mathrm{~F}$ & $0-4-9$ & 50 & 21 & 1 & 0 & 28 & 0 & 28 & 0 & 0 & 0 \\
\hline $0-5 F$ & $0-5-6$ & 64 & 18 & 13 & 0 & 33 & 0 & 33 & 0 & 0 & 0 \\
\hline
\end{tabular}

${ }^{1}$ homology-directed repair 
Table S12. $G_{3}$ progenies obtained by crossing mosaic $G_{2}$ females, derived from $y-G_{1}$ females in the presence of RU486, with wild-type males in the presence of RU486

\begin{tabular}{|c|c|c|c|c|c|c|c|c|c|c|c|}
\hline $\begin{array}{l}\text { Name } \\
\text { of } G_{1}\end{array}$ & $\begin{array}{l}\text { Name } \\
\text { of } G_{2}\end{array}$ & $\begin{array}{ll}\text { Total } & \text { number } \\
\text { of } & \mathrm{G}_{3} \\
\text { offspring }\end{array}$ & $\begin{array}{l}G_{3} \\
y- \\
j \\
\lambda\end{array}$ & $\begin{array}{l}G_{3} \\
y^{+} \\
j^{+}\end{array}$ & $\begin{array}{l}\mathrm{G}_{3} \\
\mathrm{y}- \\
0\end{array}$ & $\begin{array}{l}\mathrm{G}_{3} \\
\mathrm{y}+ \\
0 \\
+\end{array}$ & $\begin{array}{l}\text { Mosaic } \\
9 \\
+\end{array}$ & $\begin{array}{l}\text { Total } \\
\text { number } \\
\mathrm{G}_{3}+\end{array}$ & of $\begin{array}{l}\text { Total } \\
\text { number } \\
\mathrm{HDR}^{1} \text { 古 }\end{array}$ & of $\left[\begin{array}{l}\% \text { full-bodied } y \text { - } \\
\text { phenotype } G_{3} \text { 우 }\end{array}\right.$ & $\%_{3}{ }^{\circ} y$ \\
\hline $200-1 F$ & $\begin{array}{l}200-1- \\
6\end{array}$ & 48 & 19 & 5 & 0 & 24 & 0 & 24 & 0 & 0 & 0 \\
\hline $200-1 F$ & $\begin{array}{l}200-1- \\
7\end{array}$ & 31 & 11 & 0 & 9 & 3 & 8 & 20 & 17 & 45 & 85 \\
\hline $200-1 F$ & $\begin{array}{l}200-1- \\
8\end{array}$ & 47 & 20 & 3 & 8 & 6 & 10 & 24 & 18 & 33.33 & 75 \\
\hline $200-2 F$ & $200-2-$ & 48 & 10 & 11 & 0 & 27 & 0 & 27 & 0 & 0 & 0 \\
\hline $200-3 F$ & $\begin{array}{l}200-3- \\
6\end{array}$ & 52 & 18 & 5 & 0 & 29 & 0 & 29 & 0 & 0 & 0 \\
\hline $200-3 F$ & $\begin{array}{l}200-3- \\
8\end{array}$ & 36 & 7 & 7 & 0 & 22 & 0 & 22 & 0 & 0 & 0 \\
\hline $200-4 \mathrm{~F}$ & 200-4- & 46 & 19 & 1 & 10 & 10 & 6 & 26 & 16 & 38.46 & 61.54 \\
\hline $200-4 \mathrm{~F}$ & $200-4-$ & 39 & 15 & 2 & 0 & 22 & 0 & 22 & 0 & 0 & 0 \\
\hline $200-4 \mathrm{~F}$ & $\begin{array}{l}200-4- \\
8\end{array}$ & 44 & 19 & 6 & 0 & 19 & 0 & 19 & 0 & 0 & 0 \\
\hline $200-6 F$ & $\begin{array}{l}200-6- \\
6\end{array}$ & 38 & 12 & 1 & 13 & 10 & 2 & 25 & 15 & 52 & 60 \\
\hline $200-7 \mathrm{~F}$ & $\begin{array}{l}200-7- \\
6\end{array}$ & 67 & 30 & 0 & 0 & 37 & 0 & 37 & 0 & 0 & 0 \\
\hline $200-9 F$ & $\begin{array}{l}200-9- \\
6\end{array}$ & 3 & 0 & 2 & 0 & 1 & 0 & 1 & 0 & 0 & 0 \\
\hline
\end{tabular}

${ }^{1}$ homology-directed repair 
Table S13. A population-based experiment to examine the long-term effects of mutagenic chain reaction inhibition by $R U 486$

\begin{tabular}{|c|c|c|c|c|c|c|c|c|c|}
\hline $\begin{array}{l}\text { Experiment } \\
\text { number }\end{array}$ & $\begin{array}{l}\text { Total } \\
\text { number of } \\
\mathrm{G}_{3} \\
\text { offspring }\end{array}$ & $\begin{array}{c}\mathrm{G}_{3} \\
\mathrm{y}- \\
\bar{\partial}\end{array}$ & $\begin{array}{c}\mathrm{G}_{3} \\
\mathrm{y}^{+} \\
\hat{\jmath}\end{array}$ & $\begin{array}{l}\mathrm{G}_{3} \\
\mathrm{y}- \\
\mathrm{O}\end{array}$ & $\begin{array}{l}\mathrm{G}_{3} \\
\mathrm{y}^{+} \\
\dot{+}\end{array}$ & $\begin{array}{l}\text { Mosaic } \\
\text { t }\end{array}$ & $\begin{array}{l}\mathrm{RU} 486 \\
\text { concentration }\end{array}$ & $\begin{array}{l}\text { Full-bodied } \\
y \text { - } \\
\text { phenotype } \\
G_{3}\end{array}$ & $\begin{array}{l}\% \quad y- \\
\mathrm{G}_{3}\end{array}$ \\
\hline $\begin{array}{l}\text { Experiment } \\
1\end{array}$ & 358 & 55 & 112 & 13 & 172 & 6 & $0 \mu \mathrm{M}$ & 18.99 & 20.67 \\
\hline $\begin{array}{l}\text { Experiment } \\
2\end{array}$ & 708 & 66 & 260 & 55 & 319 & 8 & $0 \mu \mathrm{M}$ & 17.09 & 18.22 \\
\hline $\begin{array}{l}\text { Experiment } \\
3\end{array}$ & 462 & 67 & 137 & 53 & 191 & 14 & $0 \mu \mathrm{M}$ & 25.97 & 29.00 \\
\hline $\begin{array}{l}\text { Experiment } \\
1\end{array}$ & 367 & 37 & 104 & 18 & 194 & 14 & $200 \mu \mathrm{M}$ & 14.99 & 18.80 \\
\hline $\begin{array}{l}\text { Experiment } \\
2\end{array}$ & 433 & 15 & 154 & 9 & 253 & 2 & $200 \mu \mathrm{M}$ & 5.54 & 6.00 \\
\hline $\begin{array}{l}\text { Experiment } \\
3\end{array}$ & 467 & 45 & 112 & 57 & 239 & 14 & $200 \mu \mathrm{M}$ & 21.84 & 24.84 \\
\hline
\end{tabular}


Table S14. Population data of phenotype frequencies used for model fitting

\begin{tabular}{|c|c|c|c|c|c|}
\hline Cage & Generation & $y$ - frequency & RU486 dose & Gender & Exp. Тype \\
\hline \multirow[t]{37}{*}{$1 \mathrm{~F}$} & \multirow[t]{3}{*}{0} & 0 & 0 & Male & Closed \\
\hline & & 1 & 0 & Female & Closed \\
\hline & & 0 & 0 & Female mosaic & Closed \\
\hline & \multirow[t]{3}{*}{1} & 0.865385 & 0 & Male & Closed \\
\hline & & 0.649682 & 0 & Female & Closed \\
\hline & & 0.229299 & 0 & Female mosaic & Closed \\
\hline & \multirow[t]{3}{*}{2} & 0.704545 & 0 & Male & Closed \\
\hline & & 0.758621 & 0 & Female & Closed \\
\hline & & 0 & 0 & Female mosaic & Closed \\
\hline & \multirow[t]{3}{*}{3} & 0.732997 & 0 & Male & Closed \\
\hline & & 0.592417 & 0 & Female & Closed \\
\hline & & 0.004739 & 0 & Female mosaic & Closed \\
\hline & \multirow[t]{3}{*}{0} & 0 & 200 & Male & Closed \\
\hline & & 1 & 200 & Female & Closed \\
\hline & & 0 & 200 & Female mosaic & Closed \\
\hline & \multirow[t]{3}{*}{1} & 0.862069 & 200 & Male & Closed \\
\hline & & 0.56051 & 200 & Female & Closed \\
\hline & & 0.280255 & 200 & Female mosaic & Closed \\
\hline & \multirow[t]{3}{*}{2} & 0.671875 & 200 & Male & Closed \\
\hline & & 0.76087 & 200 & Female & Closed \\
\hline & & 0.081522 & 200 & Female mosaic & Closed \\
\hline & \multirow[t]{3}{*}{3} & 0.920152 & 200 & Male & Closed \\
\hline & & 0.828571 & 200 & Female & Closed \\
\hline & & 0.003175 & 200 & Female mosaic & Closed \\
\hline & \multirow[t]{3}{*}{0} & 0 & 0 & Male & Open \\
\hline & & 1 & 0 & Female & Open \\
\hline & & 0 & 0 & Female mosaic & Open \\
\hline & \multirow[t]{3}{*}{1} & . & 0 & Male & Open \\
\hline & & . & 0 & Female & Open \\
\hline & & . & 0 & Female mosaic & Open \\
\hline & \multirow[t]{3}{*}{2} & . & 0 & Male & Open \\
\hline & & . & 0 & Female & Open \\
\hline & & . & 0 & Female mosaic & Open \\
\hline & \multirow[t]{3}{*}{3} & 0.328431 & 0 & Male & Open \\
\hline & & 0.205426 & 0 & Female & Open \\
\hline & & 0.054264 & 0 & Female mosaic & Open \\
\hline & 0 & 0 & 200 & Male & Open \\
\hline
\end{tabular}




\begin{tabular}{|c|c|c|c|c|c|}
\hline & & 1 & 200 & Female & Open \\
\hline & & 0 & 200 & Female mosaic & Open \\
\hline & 1 & . & 200 & Male & Open \\
\hline & & . & 200 & Female & Open \\
\hline & & . & 200 & Female mosaic & Open \\
\hline & 2 & . & 200 & Male & Open \\
\hline & & . & 200 & Female & Open \\
\hline & & . & 200 & Female mosaic & Open \\
\hline & 3 & 0.286624 & 200 & Male & Open \\
\hline & & 0.183871 & 200 & Female & Open \\
\hline & & 0.045161 & 200 & Female mosaic & Open \\
\hline $2 \mathrm{~F}$ & 0 & 0 & 0 & Male & Closed \\
\hline & & 1 & 0 & Female & Closed \\
\hline & & 0 & 0 & Female mosaic & Closed \\
\hline & 1 & 0.815217 & 0 & Male & Closed \\
\hline & & 0.507042 & 0 & Female & Closed \\
\hline & & 0.309859 & 0 & Female mosaic & Closed \\
\hline & 2 & 0.646617 & 0 & Male & Closed \\
\hline & & 0.751515 & 0 & Female & Closed \\
\hline & & 0.036364 & 0 & Female mosaic & Closed \\
\hline & 3 & 0.778547 & 0 & Male & Closed \\
\hline & & 0.649057 & 0 & Female & Closed \\
\hline & & 0.049057 & 0 & Female mosaic & Closed \\
\hline & 0 & 0 & 200 & Male & Closed \\
\hline & & 1 & 200 & Female & Closed \\
\hline & & 0 & 200 & Female mosaic & Closed \\
\hline & 1 & 0.806061 & 200 & Male & Closed \\
\hline & & 0.458333 & 200 & Female & Closed \\
\hline & & 0.3125 & 200 & Female mosaic & Closed \\
\hline & 2 & 0.717593 & 200 & Male & Closed \\
\hline & & 0.833935 & 200 & Female & Closed \\
\hline & & 0.00722 & 200 & Female mosaic & Closed \\
\hline & 3 & 0.882155 & 200 & Male & Closed \\
\hline & & 0.53605 & 200 & Female & Closed \\
\hline & & 0.128527 & 200 & Female mosaic & Closed \\
\hline & 0 & 0 & 0 & Male & Open \\
\hline & & 1 & 0 & Female & Open \\
\hline & & 0 & 0 & Female mosaic & Open \\
\hline & 1 & . & 0 & Male & Open \\
\hline & & . & 0 & Female & Open \\
\hline
\end{tabular}




\begin{tabular}{|c|c|c|c|c|c|}
\hline & & & 0 & Female mosaic & Open \\
\hline & \multirow[t]{3}{*}{2} & & 0 & Male & Open \\
\hline & & & 0 & Female & Open \\
\hline & & & 0 & Female mosaic & Open \\
\hline & \multirow[t]{3}{*}{3} & 0.202454 & 0 & Male & Open \\
\hline & & 0.143979 & 0 & Female & Open \\
\hline & & 0.020942 & 0 & Female mosaic & Open \\
\hline & \multirow[t]{3}{*}{0} & 0 & 200 & Male & Open \\
\hline & & 1 & 200 & Female & Open \\
\hline & & 0 & 200 & Female mosaic & Open \\
\hline & \multirow[t]{3}{*}{1} & . & 200 & Male & Open \\
\hline & & . & 200 & Female & Open \\
\hline & & . & 200 & Female mosaic & Open \\
\hline & \multirow[t]{3}{*}{2} & . & 200 & Male & Open \\
\hline & & . & 200 & Female & Open \\
\hline & & . & 200 & Female mosaic & Open \\
\hline & \multirow[t]{3}{*}{3} & 0.088757 & 200 & Male & Open \\
\hline & & 0.034091 & 200 & Female & Open \\
\hline & & 0.007576 & 200 & Female mosaic & Open \\
\hline \multirow[t]{21}{*}{$2 \mathrm{M}$} & \multirow[t]{3}{*}{0} & 1 & 0 & Male & Closed \\
\hline & & 0 & 0 & Female & Closed \\
\hline & & 0 & 0 & Female mosaic & Closed \\
\hline & \multirow[t]{3}{*}{1} & 0 & 0 & Male & Closed \\
\hline & & 0.928571 & 0 & Female & Closed \\
\hline & & 0 & 0 & Female mosaic & Closed \\
\hline & \multirow[t]{3}{*}{2} & 0.5625 & 0 & Male & Closed \\
\hline & & 0.343284 & 0 & Female & Closed \\
\hline & & 0.099502 & 0 & Female mosaic & Closed \\
\hline & \multirow[t]{3}{*}{3} & 0.5 & 0 & Male & Closed \\
\hline & & 0.52459 & 0 & Female & Closed \\
\hline & & 0.04918 & 0 & Female mosaic & Closed \\
\hline & \multirow[t]{3}{*}{0} & 1 & 200 & Male & Closed \\
\hline & & 0 & 200 & Female & Closed \\
\hline & & 0 & 200 & Female mosaic & Closed \\
\hline & \multirow[t]{3}{*}{1} & 0 & 200 & Male & Closed \\
\hline & & 0.202899 & 200 & Female & Closed \\
\hline & & 0 & 200 & Female mosaic & Closed \\
\hline & \multirow[t]{3}{*}{2} & 0.560847 & 200 & Male & Closed \\
\hline & & 0.101266 & 200 & Female & Closed \\
\hline & & 0.042194 & 200 & Female mosaic & Closed \\
\hline
\end{tabular}




\begin{tabular}{|c|c|c|c|c|c|}
\hline & \multirow[t]{3}{*}{3} & 0.170213 & 200 & Male & Closed \\
\hline & & 0.140741 & 200 & Female & Closed \\
\hline & & 0.022222 & 200 & Female mosaic & Closed \\
\hline \multirow[t]{37}{*}{$3 \mathrm{~F}$} & \multirow[t]{3}{*}{0} & 0 & 0 & Male & Closed \\
\hline & & 1 & 0 & Female & Closed \\
\hline & & 0 & 0 & Female mosaic & Closed \\
\hline & \multirow[t]{3}{*}{1} & 0.913333 & 0 & Male & Closed \\
\hline & & 0.664835 & 0 & Female & Closed \\
\hline & & 0.230769 & 0 & Female mosaic & Closed \\
\hline & \multirow[t]{3}{*}{2} & 0.645283 & 0 & Male & Closed \\
\hline & & 0.709877 & 0 & Female & Closed \\
\hline & & 0.033951 & 0 & Female mosaic & Closed \\
\hline & \multirow[t]{3}{*}{3} & 0.869018 & 0 & Male & Closed \\
\hline & & 0.833787 & 0 & Female & Closed \\
\hline & & 0.013624 & 0 & Female mosaic & Closed \\
\hline & \multirow[t]{3}{*}{0} & 0 & 200 & Male & Closed \\
\hline & & 1 & 200 & Female & Closed \\
\hline & & 0 & 200 & Female mosaic & Closed \\
\hline & \multirow[t]{3}{*}{1} & 0.879433 & 200 & Male & Closed \\
\hline & & 0.54 & 200 & Female & Closed \\
\hline & & 0.293333 & 200 & Female mosaic & Closed \\
\hline & \multirow[t]{3}{*}{2} & 0.598513 & 200 & Male & Closed \\
\hline & & 0.614841 & 200 & Female & Closed \\
\hline & & 0.067138 & 200 & Female mosaic & Closed \\
\hline & \multirow[t]{3}{*}{3} & 0.569231 & 200 & Male & Closed \\
\hline & & 0.303846 & 200 & Female & Closed \\
\hline & & 0.011538 & 200 & Female mosaic & Closed \\
\hline & \multirow[t]{3}{*}{0} & 0 & 0 & Male & Open \\
\hline & & 1 & 0 & Female & Open \\
\hline & & 0 & 0 & Female mosaic & Open \\
\hline & \multirow[t]{3}{*}{1} & & 0 & Male & Open \\
\hline & & & 0 & Female & Open \\
\hline & & & 0 & Female mosaic & Open \\
\hline & \multirow[t]{3}{*}{2} & . & 0 & Male & Open \\
\hline & & . & 0 & Female & Open \\
\hline & & . & 0 & Female mosaic & Open \\
\hline & \multirow[t]{3}{*}{3} & 0.329341 & 0 & Male & Open \\
\hline & & 0.068063 & 0 & Female & Open \\
\hline & & 0.031414 & 0 & Female mosaic & Open \\
\hline & 0 & 0 & 200 & Male & Open \\
\hline
\end{tabular}




\begin{tabular}{|c|c|c|c|c|c|}
\hline & & 1 & 200 & Female & Open \\
\hline & & 0 & 200 & Female mosaic & Open \\
\hline & 1 & ${ }^{2}$ & 200 & Male & Open \\
\hline & & . & 200 & Female & Open \\
\hline & & . & 200 & Female mosaic & Open \\
\hline & 2 & . & 200 & Male & Open \\
\hline & & . & 200 & Female & Open \\
\hline & & . & 200 & Female mosaic & Open \\
\hline & 3 & 0.262411 & 200 & Male & Open \\
\hline & & 0.079646 & 200 & Female & Open \\
\hline & & 0.061947 & 200 & Female mosaic & Open \\
\hline $3 \mathrm{M}$ & 0 & 1 & 0 & Male & Closed \\
\hline & & 0 & 0 & Female & Closed \\
\hline & & 0 & 0 & Female mosaic & Closed \\
\hline & 1 & 0 & 0 & Male & Closed \\
\hline & & 0.570732 & 0 & Female & Closed \\
\hline & & 0 & 0 & Female mosaic & Closed \\
\hline & 2 & 0.80456 & 0 & Male & Closed \\
\hline & & 0.32381 & 0 & Female & Closed \\
\hline & & 0.295238 & 0 & Female mosaic & Closed \\
\hline & 3 & 0.68662 & 0 & Male & Closed \\
\hline & & 0.845714 & 0 & Female & Closed \\
\hline & & 0.014286 & 0 & Female mosaic & Closed \\
\hline & 0 & 1 & 200 & Male & Closed \\
\hline & & 0 & 200 & Female & Closed \\
\hline & & 0 & 200 & Female mosaic & Closed \\
\hline & 1 & 0 & 200 & Male & Closed \\
\hline & & 0.714286 & 200 & Female & Closed \\
\hline & & 0.004762 & 200 & Female mosaic & Closed \\
\hline & 2 & 0.732342 & 200 & Male & Closed \\
\hline & & 0.476703 & 200 & Female & Closed \\
\hline & & 0.290323 & 200 & Female mosaic & Closed \\
\hline & 3 & 0.440945 & 200 & Male & Closed \\
\hline & & 0.475352 & 200 & Female & Closed \\
\hline & & 0.042254 & 200 & Female mosaic & Closed \\
\hline $0-4 \mathrm{M}$ & 0 & 1 & 0 & Male & Closed \\
\hline & & 0 & 0 & Female & Closed \\
\hline & & 0 & 0 & Female mosaic & Closed \\
\hline & 1 & 0 & 0 & Male & Closed \\
\hline & & 0.736434 & 0 & Female & Closed \\
\hline
\end{tabular}




\begin{tabular}{|c|c|c|c|c|}
\hline & 0 & 0 & Female mosaic & Closed \\
\hline \multirow[t]{3}{*}{2} & 0.832797 & 0 & Male & Closed \\
\hline & 0.639752 & 0 & Female & Closed \\
\hline & 0.152174 & 0 & Female mosaic & Closed \\
\hline \multirow[t]{3}{*}{3} & 0.67147 & 0 & Male & Closed \\
\hline & 0.832386 & 0 & Female & Closed \\
\hline & 0.017045 & 0 & Female mosaic & Closed \\
\hline \multirow[t]{3}{*}{0} & 1 & 200 & Male & Closed \\
\hline & 0 & 200 & Female & Closed \\
\hline & 0 & 200 & Female mosaic & Closed \\
\hline \multirow[t]{3}{*}{1} & 0 & 200 & Male & Closed \\
\hline & 0.725275 & 200 & Female & Closed \\
\hline & 0 & 200 & Female mosaic & Closed \\
\hline \multirow[t]{3}{*}{2} & 0.732824 & 200 & Male & Closed \\
\hline & 0.475155 & 200 & Female & Closed \\
\hline & 0.242236 & 200 & Female mosaic & Closed \\
\hline \multirow[t]{3}{*}{3} & 0.805921 & 200 & Male & Closed \\
\hline & 0.802048 & 200 & Female & Closed \\
\hline & 0.020478 & 200 & Female mosaic & Closed \\
\hline
\end{tabular}




\section{The full sequences of the CGD cassette}

The full sequences of the CGD cassette are given in the following box, along with the descriptions of different sequence segments.

tttccataggctccgcccccctgacgagcatcacaaaaatcgacgctcaagtcagaggtggcgaaacccgacaggactataaagatac caggcgtttccccctggaagctccctcgtgcgctctcctgttccgaccctgccgcttaccggatacctgtccgcctttctcccttcgggaagcgt ggcgctttctcatagctcacgctgtaggtatctcagttcggtgtaggtcgttcgctccaagctgggctgtgtgcacgaaccccccgttcagccc gaccgctgcgccttatccggtaactatcgtcttgagtccaacccggtaagacacgacttatcgccactggcagcagccactggtaacagg attagcagagcgaggtatgtaggcggtgctacagagttcttgaagtggtggcctaactacggctacactagaaggacagtatttggtatctg cgctctgctgaagccagttaccttcggaaaaagagttggtagctcttgatccggcaaacaaaccaccgctggtagcggtggttttttgtttgc aagcagcagattacgcgcagaaaaaaaggatctcaagaagatcctttgatctttctacggggtctgacgctcagtggaacgaaaactca cgttaagggattttggtcatgagattatcaaaaaggatcttcacctagatcctttaaattaaaaatgaagttttaaatcaatctaaagtatatatg agtaaacttggtctgacagttaccaatgcttaatcagtgaggcacctatctcagcgatctgtctatttcgttcatccatagttgcctgactccccgt cgtgtagataactacgatacgggagggcttaccatctggccccagtgctgcaatgataccgcgagacccacgctcaccggctccagattt atcagcaataaaccagccagccggaagggccgagcgcagaagtggtcctgcaactttatccgcctccatccagtctattaattgttgccgg gaagctagagtaagtagttcgccagttaatagtttgcgcaacgttgttgccattgctacaggcatcgtggtgtcacgctcgtcgtttggtatggc ttcattcagctccggttcccaacgatcaaggcgagttacatgatcccccatgttgtgcaaaaaagcggttagctccttcggtcctccgattgttg tcagaagtaagttggccgcagtgttatcactcatggttatggcagcactgcataattctcttactgtcatgccatccgtaagatgcttttctgtgac tggtgagtactcaaccaagtcattctgagaatagtgtatgcggcgaccgagttgctcttgcccggcgtcaatacgggataataccgcgcca catagcagaactttaaaagtgctcatcattggaaaacgttcttcggggcgaaaactctcaaggatcttaccgctgttgagatccagttcgatgt aacccactcgtgcacccaactgatcttcagcatcttttactttcaccagcgtttctgggtgagcaaaaacaggaaggcaaaatgccgcaaa aaagggaataagggcgacacggaaatgttgaatactcatactcttccttttcaatattattgaagcatttatcagggttattgtctcatgagcgg atacatatttgaatgtatttagAAAAATAAACAAATAGGGGTTCCGCGCACATTTCCCCGAAAAGTGCCAC CTGACGCGCCCTGTAGCGGCGCATTAAGCGCGGCGGGTGTGGTGGTTACGCGCAGCGTGAC CGCTACACTTGCCAGCGCCCTAGCGCCCGCTCCTTTCGCTTTCTTCCCTTCCTTTCTCGCCAC GTTCGCCGGCTTTCCCCGTCAAGCTCTAAATCGGGGGCTCCCTTTAGGGTTCCGATTTAGTGC TTTACGGCACCTCGACCCCAAAAAACTTGATTAGGGTGATGGTTCACGTAGTGGGCCATCGCC CTGATAGACGGTTTTTCGCCCTTTGACGTTGGAGTCCACGTTCTTTAATAGTGGACTCTTGTTC CAAACTGGAACAACACTCAACCCTATCTCGGTCTATTCTTTTGATTTATAAGGGATTTTGCCGA TTTCGGCCTATTGGTTAAAAAATGAGCTGATTTAACAAAAATTTAACGCGAATTTTAACAAAATA TTAACGCTTACAATTTccattcgccattcaggctgcgcaactgttgggaaggggcgatCGGATTGAAAGGTGCATTA TGCTCAAATGTATATTTATCGCAACCCCCGGTTACTTTGTAAAGCAAAAACGCCTGGTTTGATT TTTAAGAAGATGGGTCGGTAAATCGATAAAAGCTATATTTTCTGGTCGTTGCAGTCTCACTCGC CTGCTATAAAAACATTAAAAGTTCCCAGAAACAATAAATGTCTTTAAATTCAATTAACGAAGAAA TAAAGAAGGAAAAGAACTGGAGCGGAAATCGGTCGAAATACTGCCAATGGCCACATATACATT TAACAGCGATATATGGTATACATATTGATAATGATGTCAGACGCAATTGCTTCAGACGGCTAAT GACATCGCAAATTGCACGCAACTTGCAATAGTGCCAATTATGACTGAAGTACATATAGCCGGG GATCTTTTAACATAAACTTCCAGTAGATGTACAAGCAGAAAAAAGAGCCATTAGCACGGCAGTT ACCATTGCTTATGATTCCTTGTGTCCAAAATAATGACAAATAGGTATATAAATAATTAAATGCCA AACATAAGCGATTCTAATTTACCTTTACATCTGTATGCATTTACATATTATCCAGAAAACAGACA GCGATAACTTGCAACATTGCTTAGTATAATAATCCAAAGAAGGAATTTAGGCAGAAATTCCAGT TAATTAAATATTCAAAACAAACTTTATTTAGTGCCTCAATAATAGTTTGGCCCTGCTAATTCTCC TATTTTATTTTTTAAGGGATTCCGGCCACTCTGACCTATATAAACATGGACCGCAGTTTGACGGG TTCACCGGAGCTAATTCCGTATCCAGATTGGCGCTCAAATACAGCTGGAGATTGCGCCAACAG TATTACCACTGCCTACCGCATTAAAGTGGATGAGTGTGGTCGGCTGTGGGTTTTGGACACTGG AACGATcggtgcgggcctcttcgctattacgccagctggcgaaagggggatgtgctgcaaggcgattaagttgggtaacgccagggtt ttcccagtcacgacgttgtaaaacgacggccagtgagcgcgcgtaatacgactcactatagggcgaattggagctccaccgcggtggcG GCCTtTCTAGgggtgccagggcgtgccettgggctccccgggcgcgtaTCTAGATTGATGAAAGAATAACGTATT CTTTCATCAAGGCGCGCCTCTGCAGAATTCGCCCTTCTGCAG CTGGTTGTAGGTGCAGTTGC GCTTCTTGCACTTGGCGCACTTGAGGAGATCGGTCTTGGTGCCCTGCACGGTGGCCAGCTGG GCACCGTTGATGGCCTCCTTGACGAACTTCTCGCGCAGCTTCTTCATCTCGTCGCTGGCCATC TCCTCCGGCGTCATTTTGGCCAGCTGCTTGGCAGTGACGGCGCCGCACATAAAGTTGCCGCG CAATCCAGGATTCTTGGGGTCCTTCAGATTGGCCACGCGTGACCTAATGCGATTCTTGTACTT CATATCCGTGTTATTGAACTCGGAGTAAATGGCATCCTCCAGTTCGGCGGCCATTTCCTCCGG CTCACCGCATCCCTCGGGCACTTCGCCAATCTTCAGTGCGGTGGCCAGCATTTCGCGGCATT TGATGCGGACCGCATCTGTCATGCCACCGGAGGGAAACGAGGTCTGTGACGAGGATGTCGA GCCTTTCTTCTCTTTATCCTTGGATGAGCTGGATGAACTGGACTTATCCTTGCCGGAGATTGAC GACGACGACTTGGCGGCACTGGTGGACTTCGAGGCACTGCTGTTGTTGGAGGAGCCCTCCTT AGCGGAGCTGTTGTTCGGTGTGGTTGGCGCCGGGCTGGCCAGGAAGCGTTTCCAGTTCTTGA 
TCAGCGTCTTGGCCAGAGCGATCACCTCGTCGTCCTTGCTACTCTTGCGCAGCTCGTTTACGG TCATGCCGATGCGCGTTTTGGTCAGAATGTCGAGATTGATGTTAAGCGTTTGCAGGGCCTTCA GCAGGTCCAGAGCCTGATCCTGTCCCTGTACGGTGGATATATGTATATTTGTGACATGATGCG CGGCACGTTAGCTAATCCCGATACTTACTGTGCCGTCGCTGGCCATCTTGCTCATCTTCTTTT GGATTCGAAACACTTCCTCCTCCACGCTCATTTTGGCCCACTTAATTTGATCAGAGGTTAGGTT AACTGGCGTCGGCTGTCGGCTTTAATCGGTGCTGCGGGTCTGCGACAGTTGTTTATGCGGAA GCCGGGCTATTTCTTTTTTTCCCTCCAATAAATGTTGTTTACTATTTTTTTCGTTTCTGTATTCGC AGAACAAGTTGACAGAACACCCTAACGCGTTTTAGTGTTACCGGGCGGTGGCTTGTCAAGAAA TACCTTCCCATGGCTAATACAATTCCCAACTGGAAGTAGTTTGGGTTTCAGAAGTCAACAGTGT TACCGTATTTTAATGTACGTCTGGCTTTTAAGCGAGTACACACACATAGCATTGCGATGTTTTTT AAACTTGCACTGCTTGCAAGCAGGGGTCCACTGTATTTCAATGAAAGAACAGCGTGTTGGGCG TTTTTGAATTATACTAATTACCTTCAATAACAATTCCCTATATATCACTTAGTTTTAATAAATAAAT CGTTTGAGTGTGGTCTAGAGATGATTTATTATGATGAAGTGCGGTTGTTGGGGACAAGCGGGA TACGTCTGCGGGTCAAATCGGCGGTGGGACATAGTGAGAGGGCAACGGCGAACAATCACCAA CGCGCCCTGGTACGCGAGAAATGTTGATATCGCACATGACCTCAGCATCCACCTTCAGTTTGA GAACAAATTAAATCAGGCACATACCAGACAACCAGGAGAACACTGAGGAGGCGTCATCAAAGA TATCTTTGGACACGTGGCATAAACAAGCCAAACTATTATCTAATCAATATTTTTATACAAATTTT GTATGTTTCCATGTTATCTTCGTTGCTCCTGTTAGTTACTACGTTTCATAGCCCTTAAATTGGTT GCTTAACGTAAAATAAAATTATATTGTATAAAAATAAAGAGGAATTCACCTATAACAAACGAAAA AGTAAATAAATAAGAACTTCCCCATGTTAAAAATGCCACCACCATCTTTACATATAAGTATGTAC ATATGAATGAATCACTTAGGTTGCTTGAATATTACAATTTCATTAATAGCTAAATCACATTTGAT GTGTTAGTGGAAAACGGCTATATATATAAATTATCGAAATTGTGAATATCGAATTGCGATAGCA CAATGGGAAATTCCACCACTAGATTTTTGGTACTTTTAACAGATCCTTTTCGGTTTTGCGTTGC GCGAAGTGATCTGAACTTATCAAAGTTTGTAAGGTAATACATAAAGTGAAAAAGAATTAATTTG CTCTTGAAAGGCAGGCCAAATTAAAAAAAAAATATCAATCGGTACCGCTAGCAGATCCGCCCG CCAAGCTTGAAGCAAGCCTCAATCAAAATGAAGCTGCTGAGTAGTATTGAACAAGCGTGTGAC ATATGCCGACTTAAGAAGCTGAAGTGCAGCAAGGAAAAGCCGAAGTGCGCCAAATGTCTTAAG AACAATTGGGAGTGCCGGTACTCGCCAAAGACGAAGCGAAGCCCTTTGACACGCGCTCATTT GACTGAAGTGGAGAGTCGCCTGGAGCGCCTTGAGCAGTTGTTCCTCCTCATTTTTCCCCGTGA AGACCTCGACATGATACTGAAGATGGATAGTCTCCAAGATATTAAGGCCCTGCTCACTGGCCT GTTTGTTCAGGATAACGTCAACAAGGATGCCGTGACGGACCGCCTTGCCTCGGTGGAGACAG ATATGCCACTTACCTTGCGGCAGCATCGCATCTCGGCTACCAGCAGTAGTGAAGAAAGTTCCA ATAAGGGTCAACGGCAACTGACGGTATCGGGATCCAAGAAGTTTAATAAGGTACGAGTAGTTO GCGCCCTGGATGCAGTTGCCCTCCCGCAGCCCGTGGGCGTTCCGAACGAGAGTCAGGCCCT GAGTCAGCGGTTCACCTTTTCGCCTGGCCAAGATATTCAGTTGATCCCTCCTCTCATCAATCT GCTGATGTCGATTGAGCCTGATGTGATTTACGCGGGACACGATAACACGAAGCCTGATACAAG TTCCAGCCTGTTGACGAGCCTGAACCAGCTGGGTGAACGCCAACTGCTGTCCGTAGTCAAGT GGTCCAAGTCGCTGCCCGGCTTCAGGAATCTTCATATAGACGACCAGATCACTCTGATTCAAT ACAGTTGGATGAGTCTCATGGTGTTCGGTTTGGGTTGGCGTTCGTACAAGCATGTGAGCGGT CAAATGCTGTACTTCGCTCCCGATCTGATTCTGAATGAGCAGCGGATGAAGGAGTCCAGTTTT TACAGTCTGTGCCTGACCATGTGGCAGATCCCACAGGAGTTCGTGAAGCTGCAGGTGTCCCA AGAAGAGTTTCTGTGTATGAAGGTCCTCTTGTTGCTCAATACCATTCCTCTTGAGGGCCTGCG GAGCCAAACCCAGTTTGAGGAGATGCGTAGTTCGTATATACGGGAACTGATTAAGGCGATTGG ACTCCGCCAAAAGGGCGTGGTGTCCTCGTCGCAACGATTCTACCAACTGACGAAGCTCCTCG ATAACCTGCATGATCTTGTTAAGCAGCTCCATTTGTATTGCCTCAACACCTTTATCCAGAGCCG AGCCCTGAGCGTCGAGTTTCCTGAAATGATGAGTGAAGTAATCGCTGGTAGTACGCCGATGG AGTTCCAGTACCTGCCCGATACGGATGACCGTCACCGTATCGAAGAAAAGCGGAAGCGAACC TATGAAACCTTCAAGTCCATCATGAAAAAGTCCCCCTTCTCGGGCCCCACGGACCCGCGCCC CCCGCCCCGTCGTATTGCGGTTCCTTCGCGCAGCAGTGCCAGCGTCCCCAAACCCGCACCG CAGCCCTACCCGTTCACTTCCTCCCTTAGCACGATTAACTATGATGAGTTCCCCACGATGGTG TTCCCCAGTGGACAAATTTCCCAGGCATCGGCACTGGCTCCGGCCCCACCGCAAGTCCTCCC CCAGGCGCCCGCTCCGGCACCGGCTCCCGCAATGGTGAGTGCTCTGGCCCAGGCCCCCGCT CCAGTCCCCGTGCTGGCGCCTGGACCCCCACAGGCAGTTGCCCCTCCTGCTCCGAAACCAA CGCAGGCGGGCGAAGGAACCCTGAGCGAGGCCCTCTTGCAGCTTCAGTTCGATGACGAAGA CTTGGGAGCCCTGCTGGGTAACAGCACAGACCCTGCCGTATTCACCGATCTCGCATCCGTGG ACAACAGCGAGTTTCAGCAGCTCTTGAATCAGGGAATCCCGGTCGCACCTCATACCACAGAG CCCATGCTGATGGAATACCCGGAGGCTATCACGCGACTGGTGACCGGCGCACAGCGACCAC CCGATCCAGCCCCTGCCCCACTGGGTGCCCCGGGTTTGCCCAATGGCCTCCTCAGCGGCGA TGAGGATTTCTCCAGCATCGCTGATATGGATTTCTCCGCTTTGCTGAGCCAGATAAGCTCCTA 
AGTTTAAACAAGCTTATCGATACCGTCGACTAAAGCCAAATAGAAAATTATTCAGTTCCTGGCT TAAGTTTTTAAAAGTGATATTATTTATTTGGTTGTAACCAACCAAAAGAATGTAAATAACTAATAC ATAATTATGTTAGTTTTAAGTTAGCAACAAATTGATTTTAGCTATATTAGCTACTTGGTTAATAAA TAGAATATATTTATTTAAAGATAATTGCGTTTTTATTGTCAGGGAGTGAGTTTGCTTAAAAACTC GTTTAGATCCACTAGTggatctggatcctctagagtcgacctcgaacgttaacgttaacgtaacgttaacTCGACTAGCTC GCCAAGCAGAGAGGGCGCCAGTGCTCACTACTTTTTATAATTCTCAACTTCTTTTTCCAGACTC AGTTCGTATATATAGACCTATTTTCAATTTAACGTCGGTTTTGGACACTGGAACCGGTTTTAGA GCTAGAAATAGCAAGTTAAAATAAGGCTAGTCCGTTATCAACTTGAAAAAGTGGCACCGAGTC GGTGCTTTTTTGCCTACCTGGAGCCTGAGAGTTGTTCCTCGAGCGGCCGCCAGTGTGATGGA TATCTGCAGAATTCGCCCTTCTGCAGCTGGTTGTAGGTGCAGTTGCGCTTCTTGCACTTGGCG CACTTGAGGAGATCGGTCTTGGTGCCCTGCACGGTGGCCAGCTGGGCACCGTTGATGGCCT CCTTGACGAACTTCTCGCGCAGCTTCTTCATCTCGTCGCTGGCCATCTCCTCCGGCGTCATTT TGGCCAGCTGCTTGGCAGTGACGGCGCCGCACATAAAGTTGCCGCGCAATCCAGGATTCTTG GGGTCCTTCAGATTGGCCACGCGTGACCTAATGCGATTCTTGTACTTCATATCCGTGTTATTGA ACTCGGAGTAAATGGCATCCTCCAGTTCGGCGGCCATTTCCTCCGGCTCACCGCATCCCTCG GGCACTTCGCCAATCTTCAGTGCGGTGGCCAGCATTTCGCGGCATTTGATGCGGACCGCATC TGTCATGCCACCGGAGGGAAACGAGGTCTGTGACGAGGATGTCGAGCCTTTCTTCTCTTTATC CTTGGATGAGCTGGATGAACTGGACTTATCCTTGCCGGAGATTGACGACGACGACTTGGCGG CACTGGTGGACTTCGAGGCACTGCTGTTGTTGGAGGAGCCCTCCTTAGCGGAGCTGTTGTTC GGTGTGGTTGGCGCCGGGCTGGCCAGGAAGCGTTTCCAGTTCTTGATCAGCGTCTTGGCCAG AGCGATCACCTCGTCGTCCTTGCTACTCTTGCGCAGCTCGTTTACGGTCATGCCGATGCGCGT TTTGGTCAGAATGTCGAGATTGATGTTAAGCGTTTGCAGGGCCTTCAGCAGGTCCAGAGCCTG ATCCTGTCCCTGTACGGTGGATATATGTATATTTGTGACATGATGCGCGGCACGTTAGCTAAT CCCGATACTTACTGTGCCGTCGCTGGCCATCTTGCTCATCTTCTTTTGGATTCGAAACACTTCC TCCTCCACGCTCATTTTGGCCCACTTAATTTGATCAGAGGTTAGGTTAACTGGCGTCGGCTGT CGGCTTTAATCGGTGCTGCGGGTCTGCGACAGTTGTTTATGCGGAAGCCGGGCTATTTCTTTT TTTCCCTCCAATAAATGTTGTTTACTATTTTTTTCGTTTCTGTATTCGCAGAACAAGTTGACAGA ACACCCTAACGCGTTTTAGTGTTACCGGGCGGTGGCTTGTCAAGAAATACCTTCCCATGGCTA ATACAATTCCCAACTGGAAGTAGTTTGGGTTTCAGAAGTCAACAGTGTTACCGTATTTTAATGT ACGTCTGGCTTTTAAGCGAGTACACACACATAGCATTGCGATGTTTTTTAAACTTGCACTGCTT GCAAGCAGGGGTCCACTGTATTTCAATGAAAGAACAGCGTGTTGGGCGTTTTTGAATTATACT AATTACCTTCAATAACAATTCCCTATATATCACTTAGTTTTAATAAATAAATCGTTTGAGTGTGGT CTAGAGATGATTTATTATGATGAAGTGCGGTTGTTGGGGACAAGCGGGATACGTCTGCGGGT CAAATCGGCGGTGGGACATAGTGAGAGGGCAACGGCGAACAATCACCAACGCGCCCTGGTA CGCGAGAAATGTTGATATCGCACATGACCTCAGCATCCACCTTCAGTTTGAGAACAAATTAAAT CAGGCACATACCAGACAACCAGGAGAACACTGAGGAGGCGTCATCAAAGATATCTTTGGACA CGTGGCATAAACAAGCCAAACTATTATCTAATCAATATTTTTATACAAATTTTGTATGTTTCCAT GTTATCTTCGTTGCTCCTGTTAGTTACTACGTTTCATAGCCCTTAAATTGGTTGCTTAACGTAAA ATAAAATTATATTGTATAAAAATAAAGAGGAATTCACCTATAACAAACGAAAAAGTAAATAAATA AGAACTTCCCCATGTTAAAAATGCCACCACCATCTTTACATATAAGTATGTACATATGAATGAAT CACTTAGGTTGCTTGAATATTACAATTTCATTAATAGCTAAATCACATTTGATGTGTTAGTGGAA AACGGCTATATATATAAATTATCGAAATTGTGAATATCGAATTGCGATAGCACAATGGGAAATT CCACCACTAGATTTTTGGTACTTTTAACAGATCCTTTTCGGTTTTGCGTTGCGCGAAGTGATCT GAACTTATCAAAGTTTGTAAGGTAATACATAAAGTGAAAAAGAATTAATTTGCTCTTGAAAGGCA GGCCAAATTAAAAAAAAAATATCAATCGGTACCAGATCTGGCGCGCCTAGAATGGACTATAAG GACCACGACGGAGACTACAAGGATCATGATATTGATTACAAAGACGATGACGATAAGATGGCC CCAAAGAAGAAGCGGAAGGTCGGTATCCACGGAGTCCCAGCAGCCGACAAGAAGTACAGCAT CGGCCTGGACATCGGCACCAACTCTGTGGGCTGGGCCGTGATCACCGACGAGTACAAGGTG CCCAGCAAGAAATTCAAGGTGCTGGGCAACACCGACCGGCACAGCATCAAGAAGAACCTGAT CGGAGCCCTGCTGTTCGACAGCGGCGAAACAGCCGAGGCCACCCGGCTGAAGAGAACCGCC AGAAGAAGATACACCAGACGGAAGAACCGGATCTGCTATCTGCAAGAGATCTTCAGCAACGA GATGGCCAAGGTGGACGACAGCTTCTTCCACAGGCTGGAAGAGTCCTTCCTGGTGGAAGAGG ATAAGAAGCACGAGCGGCACCCCATCTTCGGCAACATCGTGGACGAGGTGGCCTACCACGAG AAGTACCCCACCATCTACCACCTGAGAAAGAAACTGGTGGACAGCACCGACAAGGCCGACCT GCGGCTGATCTATCTGGCCCTGGCCCACATGATCAAGTTCCGGGGCCACTTCCTGATCGAGG GCGACCTGAACCCCGACAACAGCGACGTGGACAAGCTGTTCATCCAGCTGGTGCAGACCTAC AACCAGCTGTTCGAGGAAAACCCCATCAACGCCAGCGGCGTGGACGCCAAGGCCATCCTGTC TGCCAGACTGAGCAAGAGCAGACGGCTGGAAAATCTGATCGCCCAGCTGCCCGGCGAGAAG AAGAATGGCCTGTTCGGAAACCTGATTGCCCTGAGCCTGGGCCTGACCCCCAACTTCAAGAG 
CAACTTCGACCTGGCCGAGGATGCCAAACTGCAGCTGAGCAAGGACACCTACGACGACGACC TGGACAACCTGCTGGCCCAGATCGGCGACCAGTACGCCGACCTGTTTCTGGCCGCCAAGAAC CTGTCCGACGCCATCCTGCTGAGCGACATCCTGAGAGTGAACACCGAGATCACCAAGGCCCC CCTGAGCGCCTCTATGATCAAGAGATACGACGAGCACCACCAGGACCTGACCCTGCTGAAAG CTCTCGTGCGGCAGCAGCTGCCTGAGAAGTACAAAGAGATTTTCTTCGACCAGAGCAAGAAC GGCTACGCCGGCTACATTGACGGCGGAGCCAGCCAGGAAGAGTTCTACAAGTTCATCAAGCC CATCCTGGAAAAGATGGACGGCACCGAGGAACTGCTCGTGAACAGAGAGGACCTGCTGCGG AAGCAGCGGACCTTCGACAACGGCAGCATCCCCCACCAGATCCACCTGGGAGAGCTGCACG CCATTCTGCGGCGGCAGGAAGATTTTTACCCATTCCTGAAGGACAACCGGGAAAAGATCGAG AAGATCCTGACCTTCCGCATCCCCTACTACGTGGGCCCTCTGGCCAGGGGAAACAGCAGATT CGCCTGGATGACCAGAAAGAGCGAGGAAACCATCACCCCCTGGAACTTCGAGGAAGTGGTG GACAAGGGCGCTTCCGCCCAGAGCTTCATCGAGCGGATGACCAACTTCGATAAGAACCTGCC CAACGAGAAGGTGCTGCCCAAGCACAGCCTGCTGTACGAGTACTTCACCGTGTATAACGAGC TGACCAAAGTGAAATACGTGACCGAGGGAATGAGAAAGCCCGCCTTCCTGAGCGGCGAGCAG AAAAAGGCCATCGTGGACCTGCTGTTCAAGACCAACCGGAAAGTGACCGTGAAGCAGCTGAA AGAGGACTACTTCAAGAAAATCGAGTGCTTCGACTCCGTGGAAATCTCCGGCGTGGAAGATC GGTTCAACGCCTCCCTGGGCACATACCACGATCTGCTGAAAATTATCAAGGACAAGGACTTCC TGGACAATGAGGAAAACGAGGACATTCTGGAAGATATCGTGCTGACCCTGACACTGTTTGAGG ACAGAGAGATGATCGAGGAACGGCTGAAAACCTATGCCCACCTGTTCGACGACAAAGTGATG AAGCAGCTGAAGCGGCGGAGATACACCGGCTGGGGCAGGCTGAGCCGGAAGCTGATCAACG GCATCCGGGACAAGCAGTCCGGCAAGACAATCCTGGATTTCCTGAAGTCCGACGGCTTCGCC AACAGAAACTTCATGCAGCTGATCCACGACGACAGCCTGACCTTTAAAGAGGACATCCAGAAA GCCCAGGTGTCCGGCCAGGGCGATAGCCTGCACGAGCACATTGCCAATCTGGCCGGCAGCC CCGCCATTAAGAAGGGCATCCTGCAGACAGTGAAGGTGGTGGACGAGCTCGTGAAAGTGATG GGCCGGCACAAGCCCGAGAACATCGTGATCGAAATGGCCAGAGAGAACCAGACCACCCAGA AGGGACAGAAGAACAGCCGCGAGAGAATGAAGCGGATCGAAGAGGGCATCAAAGAGCTGGG CAGCCAGATCCTGAAAGAACACCCCGTGGAAAACACCCAGCTGCAGAACGAGAAGCTGTACC TGTACTACCTGCAGAATGGGCGGGATATGTACGTGGACCAGGAACTGGACATCAACCGGCTG TCCGACTACGATGTGGACCATATCGTGCCTCAGAGCTTTCTGAAGGACGACTCCATCGACAAC AAGGTGCTGACCAGAAGCGACAAGAACCGGGGCAAGAGCGACAACGTGCCCTCCGAAGAGG TCGTGAAGAAGATGAAGAACTACTGGCGGCAGCTGCTGAACGCCAAGCTGATTACCCAGAGA AAGTTCGACAATCTGACCAAGGCCGAGAGAGGCGGCCTGAGCGAACTGGATAAGGCCGGCT TCATCAAGAGACAGCTGGTGGAAACCCGGCAGATCACAAAGCACGTGGCACAGATCCTGGAC TCCCGGATGAACACTAAGTACGACGAGAATGACAAGCTGATCCGGGAAGTGAAAGTGATCAC CCTGAAGTCCAAGCTGGTGTCCGATTTCCGGAAGGATTTCCAGTTTTACAAAGTGCGCGAGAT CAACAACTACCACCACGCCCACGACGCCTACCTGAACGCCGTCGTGGGAACCGCCCTGATCA AAAAGTACCCTAAGCTGGAAAGCGAGTTCGTGTACGGCGACTACAAGGTGTACGACGTGCGG AAGATGATCGCCAAGAGCGAGCAGGAAATCGGCAAGGCTACCGCCAAGTACTTCTTCTACAG CAACATCATGAACTTTTTCAAGACCGAGATTACCCTGGCCAACGGCGAGATCCGGAAGCGGC CTCTGATCGAGACAAACGGCGAAACCGGGGAGATCGTGTGGGATAAGGGCCGGGATTTTGC CACCGTGCGGAAAGTGCTGAGCATGCCCCAAGTGAATATCGTGAAAAAGACCGAGGTGCAGA CAGGCGGCTTCAGCAAAGAGTCTATCCTGCCCAAGAGGAACAGCGATAAGCTGATCGCCAGA AAGAAGGACTGGGACCCTAAGAAGTACGGCGGCTTCGACAGCCCCACCGTGGCCTATTCTGT GCTGGTGGTGGCCAAAGTGGAAAAGGGCAAGTCCAAGAAACTGAAGAGTGTGAAAGAGCTGC TGGGGATCACCATCATGGAAAGAAGCAGCTTCGAGAAGAATCCCATCGACTTTCTGGAAGCCA AGGGCTACAAAGAAGTGAAAAAGGACCTGATCATCAAGCTGCCTAAGTACTCCCTGTTCGAGC TGGAAAACGGCCGGAAGAGAATGCTGGCCTCTGCCGGCGAACTGCAGAAGGGAAACGAACT GGCCCTGCCCTCCAAATATGTGAACTTCCTGTACCTGGCCAGCCACTATGAGAAGCTGAAGG GCTCCCCCGAGGATAATGAGCAGAAACAGCTGTTTGTGGAACAGCACAAGCACTACCTGGAC GAGATCATCGAGCAGATCAGCGAGTTCTCCAAGAGAGTGATCCTGGCCGACGCTAATCTGGA CAAAGTGCTGTCCGCCTACAACAAGCACCGGGATAAGCCCATCAGAGAGCAGGCCGAGAATA TCATCCACCTGTTTACCCTGACCAATCTGGGAGCCCCTGCCGCCTTCAAGTACTTTGACACCA CCATCGACCGGAAGAGGTACACCAGCACCAAAGAGGTGCTGGACGCCACCCTGATCCACCA GAGCATCACCGGCCTGTACGAGACACGGATCGACCTGTCTCAGCTGGGAGGCGACAAAAGG CCGGCGGCCACGAAAAAGGCCGGCCAGGCAAAAAAGAAAAAGTAATCTAGGCCTGGAGACTC TAGCACTAGTGCATGCCTGCAGGTCGGAGTACTGTCCTCCGAGCGGAGTACTGTCCTCCGAG CGGAGACTCTAGCGACGTCGAGCGCCGGAGTATAAATAGAGGCGCTTCGTCTACGGAGCGA CAATTCAATTCAAACAAGCAAAGTGAACACGTCGCTAAGCGAAAGCTAAGCAAATAAACAAGC GCAGCTGAACAAGCTAAACAATCTGCAGTAAAGTGCAAGTTAAAGTGAATCAATTAAAAGTAAC 
CAGCAACCAAGTAAATCAACTGCAACTACTGAAATCTGCCAAGAAGTAATTATTGAATACAAGA AGAGAACTCTGAATAGATCTAAAAGGTAGGTTCAACCACTGATGCCTAGGCACACCGAAACGA CTAACCCTAATTCTTATCCTTTACTTCAGGCGGCCGCGGCTCGAGAATCAAAATGCAGCTTAC CAAGGACACTGAGATTTCCACTATAAACCGACAGATGTCCGACTTCTCCGAACTGAGTCAGAT TCTCCCCCTGCACCAGATCAGCAAGATTAAGGACATTCTGGAGAACGAGAACCCACTGCCGA AGGAAAAGCTCGCCAGCCACCTGACTATGATTATTCTTATGGCTAACCTGGCATCGCAAAAGC GTAAAGATGTGCCGGTTAAGCGCTCGACATTCCTTAAGTATCAGAGGTCCATCAGCAAGACGC TCCAATACGATTCCTCGACAAAAACAGTCTCGTTCGAGTACCACCTTAAGGACCCGAGCAAAT TGATCAAGGGCCTTGAGGACGTTGTGAGTCCGTACCGTTTTGTCGTGGGTGTGCATGAGAAG CCGGACGATGTGATGAGCCATCTTAGCGCCGTACACATGCGTAAGGAGGCAGGCCGCAAGC GTGATCTCGGCAACAAGATCAATGACGAGATAACGAAAATCGCGGAGACCCAGGAGACGATC TGGGGATTTGTCGGAAAGACCATGGATCTCATTGAAGCGCGGACTACACGGCCAACCACAAA AGCTGCCTACAATCTGCTCCTGCAAGCCACTTTCATGAACTGCTGTCGCGCCGATGACCTTAA AAACACAGACATCAAGACATTTGAGGTCATTCCGGACAAGCACTTGGGACGGATGCTCCGAG CGTTTGTCCCGGAGACGAAGACAGGCACCCGCTTTGTGTACTTCTTTCCTTGCAAAGGACGGT GCGATCCGCTGCTGGCTCTGGACAGTTACCTGCAGTGGACTGATCCGATCCCTAAAACACGC ACCACGGATGAGGATGCCCGCTATGACTACCAACTGTTGCGTAACTCGTTGCTGGGTAGCTAT GATGGTTTCATTAGTAAGCAGTCCGACGAATCGATATTCAAGATTCCCAATGGTCCGAAGGCG CACCTGGGTCGCCACGTGACGGCATCCTATTTGAGCAACAATGAGATGGACAAGGAGGCAAC ATTGTATGGAAACTGGTCGGCAGCCCGCGAAGAAGGTGTCAGCAGGGTCGCTAAAGCGCGCT ACATGCATACCATTGAGAAGTCGCCTCCAAGCTACCTTTTTGCTTTTCTGTCCGGATTTTACAA CATTACCGCCGAGCGAGCGTGCGAACTGGTGGACCCAAACTCCAACCCCTGTGAGCAAGACA AGAATATACCAATGATAAGCGATATAGAGACACTTATGGCACGTTACGGAAAGAATGCAGAAA TCATCCCGATGGATGTGCTGGTCTTCTTGAGCTCGTACGCAAGGTTTAAGAACAACGAGGGTA AGGAATATAAGCTGCAAGCTCGGTCGAGCCGCGGAGTGCCAGATTTTCCAGATAACGGACGA ACAGCGCTCTATAACGCCCTGACTGCGGCCCATGTTAAGCGCAGGAAAATCTCGATAGTCGTA GGACGTTCCATCGACACCTCGGGATCCCATGGCTTCCCTCCAGAGGTGGAGGAGCAAGATGA TGGCACTCTCCCCATGAGCTGCGCTCAAGAGAGTGGCATGGATCGTCACCCCGCTGCTTGCG CCTCGGCTCGCATCAACGTGTAATCTAGAGGATCTTTGTGAAGGAACCTTACTTCTGTGGTGT GACATAATTGGACAAACTACCTACAGAGATTTAAAGCTCTAAGGTAAATATAAAATTTTTAAGTG TATAATGTGTTAAACTACTGATTCTAATTGTTTGTGTATTTTAGATTCCAACCTATGGAACTGAT GAATGGGAGCAGTGGTGGAATGCCTTTAATGAGGAAAACCTGTTTTGCTCAGAAGAAATGCCA TCTAGTGATGATGAGGCTACTGCTGACTCTCAACATTCTACTCCTCCAAAAAAGAAGAGAAAG GTAGAAGACCCCAAGGACTTTCCTTCAGAATTGCTAAGTTTTTTGAGTCATGCTGTGTTTAGTA ATAGAACTCTTGCTTGCTTTGCTATTTACACCACAAAGGAAAAAGCTGCACTGCTATACAAGAA AATTATGGAAAAATATTTGATGTATAGTGCCTTGACTAGAGATCATAATCAGCCATACCACATTT GTAGAGGTTTTACTTGCTTTAAAAAACCTCCCACACCTCCCCCTGAACCTGAAACATAAAATGA ATGCAATTGTTGTTGTTAACTTGTTTATTGGAGCTTATAATGGTTACAAATAAAGCAATAGCATC ACAAATTTCACAAATAAAGCATTTTTTTTCACTGCATTCTAGTTGTGGTTTTGTCCAAACTCATCAA TGTATCTTATCATGTCTGGATCGATCTGGCCGGCCGTTTAAACGAATTTAATTAAACGCGTCAT ATGTTGATGAAAGAATAACGTATTCTTTCATCAAAAGCTTATTTAAATTCGCGAGCTCTTCCgctta cgcgcccggggagcccaagggcacgccctggcacccgcttcctcgctcactgactcgctgcgctcggtcgttcggctgcggcgagcggt atcagctcactcaaaggcggtaatacggttatccacagaatcaggggataacgcaggaaagaaCATGTCCGTGGGCATCG GCAATACCACCACTAATCCGTGCCCCTATGCGGTAAATGTCTTTGACTTGACCACGGATACGC GAATTCGGAGATACGAGCTACCTGGCGTGGACACAAATCCAAATACTTTCATAGCTAACATTG CCGTGGATATAGGCAAAAATTGCGATGATGCATATGCCTATTTTGCCGATGAATTGGGATACG GCTTGATTGCTTACTCCTGGGAACTGAACAAGTCCTGGAGATTCTCGGCACATTCGTATTTTTT CCCCGATCCATTGAGGGGCGATTTCAATGTCGCTGGTATTAACTTCCAATGGGGCGAGGAGG GTATATTTGGTATGTCCCTTTCGCCCATTCGATCGGATGGTTATCGTACCCTGTACTTTAGTCC GTTAGCAAGTCATCGACAATTTGCCGTATCCACGAGGATTTTGAGGGATGAAACCAGGACGGA AGATAGCTATCATGACTTTGTTGCCTTAGATGAACGGGGTCCAAACTCCCATACCACTTCACGT GTGATGAGCGATGATGGAATTGAGCTGTTCAATTTAATAGATCAAAATGCAGTGGGTTGCTGG CACTCATCAATGCCGTACTCACCGCAATTTCATGGCATTGTGGATCGCGATGACGTTGGCTTA GTTTTTCCGGCCGATGTGAAAATTGATGAGAACAAAAACGTTTGGGTTCTATCCGATAGGATG CCCGTTTTCTTGCTGTCTGACTTGGATTATTCAGATACTAATTTCCGAATTTACACGGCTCCCTT GGCCACTTTAATTGAGAATACTGTGTGTGATTTGAGGAATAACGCCTATGGGCCGCCAAATAC CGTTTCAATACCAAAACAAGCCGTTTTGCCAATGGGTCCACCGTTATATACGAAACAATATCGT CCTGTCTTGCCACAGAAACCTCAGACCAGCTGGGCTTCCTCGCCGCCTCCTCCAAGTCGCAC 
TTATTTGCCCGCCAATTCAGGCAATGTAGTCTCCAGAcatgtgagcaaaaggccagcaaaaggccaggaacc gtaaaaaggccgcgttgctggcgtt

HA1; homology arm 1 - in red

RRS; Rippase recombinase site - in dark blue

Vasa-Gene Switch - in yellow

U6-y-sgRNA - in green

Vasa-Cas9 - in blue

2X UAS-Rippase - in purple

HA2; homology arm 2 - in red 


\section{Supplemental references}

1. Gantz, V. M.; Bier, E., Genome editing. The mutagenic chain reaction: a method for converting heterozygous to homozygous mutations. Science 2015, 348 (6233), 442-4. 